\title{
A One-Pot Biginelli Synthesis and Characterization of Novel Dihydropyrimidinone Derivatives Containing Piperazine/Morpholine Moiety
}

\author{
Mashooq Ahmad Bhat ${ }^{1, *(1)}$, Mohamed A. Al-Omar ${ }^{1}$ (D), Hazem A. Ghabbour ${ }^{1,2}$ (D) \\ and Ahmed M. Naglah ${ }^{3,4}$ \\ 1 Department of Pharmaceutical Chemistry, College of Pharmacy, King Saud University, Riyadh 11451, \\ Saudi Arabia; malomar1@ksu.edu.sa (M.A.A.-O.); ghabbourh@yahoo.com (H.A.G.) \\ 2 Department of Medicinal Chemistry, Faculty of Pharmacy, University of Mansoura, Mansoura 35516, Egypt \\ 3 Department of Pharmaceutical Chemistry, Drug Exploration and Development Chair (DEDC), \\ College of Pharmacy, King Saud University, Riyadh 11451, Saudi Arabia; amnaglah@gmail.com \\ 4 Peptide Chemistry Department, Chemical Industries Research Division, National Research Centre, Dokki, \\ Cairo 12622, Egypt \\ * Correspondence: mabhat@ksu.edu.sa; Tel.: +966-55-816-4097
}

Received: 30 May 2018; Accepted: 21 June 2018; Published: 27 June 2018

\begin{abstract}
Enaminones, 4-methyl-1-[4-(piperazin/morpholin-1-yl) phenyl] pent-2-en-1-one (IIa-b) were synthesized by refluxing 1-[4-(piperazin/morpholin-1-yl) phenyl] ethan-1-one (Ia-b) with dimethylformamide dimethylacetal (DMF-DMA) without any solvent. The three dimensional structure of enaminone (IIb) containing morpholine moiety was confirmed by single crystal X-ray crystallography. Finally, the dihydropyrimidinone derivatives (1-20) were obtained by reacting enaminones (IIa-b) with urea and different substituted benzaldehydes in the presence of glacial acetic acid. Dihydropyrimidinone derivatives containing piperazine/morpholine moiety were synthesized in a good yield by means of simple and efficient method.
\end{abstract}

Keywords: dihydropyrimidinone derivatives; morpholine; piperazine; Biginelli synthesis

\section{Introduction}

Pyrimidines scaffold have played a significant role in the area of medicinal chemistry [1]. Pyrimidines are important moieties because of their potential biological activities such as antitumor, antiviral, and antibacterial agents [2,3]. Dihydropyridines are the most potent calcium channel modulators available for the treatment of various cardiovascular diseases [4]. Dihydropyrimidines, also known as Biginelli's compounds, display various pharmacological activities [5]. Dihydropyrimidinone compounds were first synthesized by Pietro Biginelli. The process comprised of reacting numerous aldehydes with urea and a beta-keto ester to give a tetrahydropyrimidinone. Substituted dihydropyrimidinone compounds show interesting biological properties. Some of the analogs of dihydropyrimidine compounds are antitumor agents [6]. Dihydropyrimidinones have emerged as calcium channel blockers and antihypertensive agents [7]. These compounds exhibit a broad range of pharmacological activities, such as antibacterial, antitumor, antiviral, and anti-inflammatory [8].

Piperazine moiety contains two nitrogen atoms at two opposite positions of a six-membered heterocyclic ring. Polar nitrogen atoms increase the favorable interactions of piperazine with macromolecules. It has the ability to cross the blood brain barrier (BBB) due to its lipophilic nature, and is useful in various diseases, such as Alzheimer's disease, psychosis, and depression. Many potent marketed drugs like fluphenazine, cinnarizine, flunarizine, lomerizine, ciprofloxacin, indinavir, etc., have a piperazine moiety (Figure 1). Piperazine derivatives have shown significant pharmacological 
activities, such as anti-tuberculosis, anti-inflammatory, antiviral, as Central Nervous System (CNS) agents, anticancer, as cardioprotective agents, and antidiabetic [9-28].
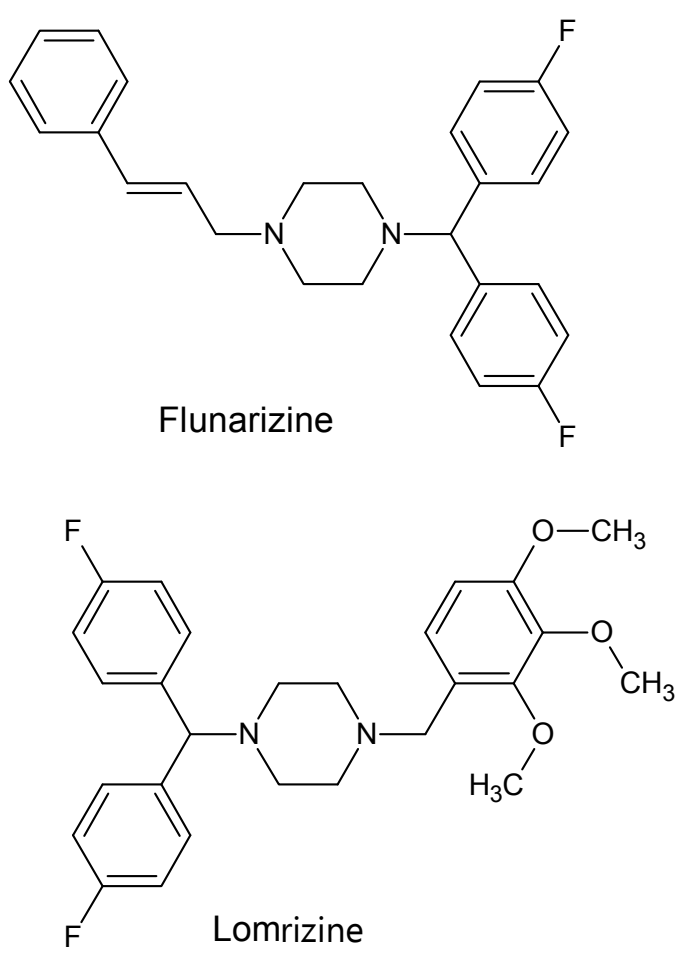<smiles>O=C(O)c1cn(C2CC2)c2cc(N3CCNCC3)c(F)cc2c1=O</smiles>

Ciprofloxacin

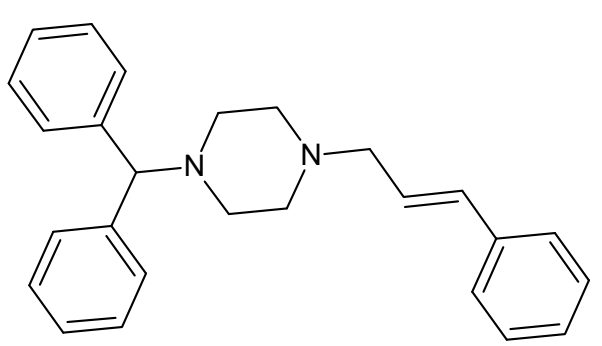

Cinnarizine<smiles>CC(C)(C)NC(=O)[C@H]1CN(Cc2cccnc2)CCN1C[C@H](O)C[C@H](Cc1ccccc1)C(=O)N[C@H]1c2ccccc2C[C@H]1O</smiles>

Figure 1. Marketed drugs containing piperazine moiety.

Morpholine is an organic moiety containing nitrogen and oxygen in a heterocyclic six-membered ring, and is considered as an important building block in the field of medicinal chemistry. The linezolid antibiotic having a morpholine moiety is commercially available as antimicrobial agent. Timolol, moclobemide, emorfazone (anti-inflammatory drug and analgesic), phenadoxone (heptalgin, opioid analgesic), antidepressants reboxetine and gefitinib, fenpropimorph (fungicide) and antibacterial drugs finafloxacin and levofloxacin contain a morpholine moiety (Figure 2). Morpholine derivatives are very much essential in the drug discovery process. Morpholine scaffolds are important, due to their variety of pharmacological activities [29-35].

The literature review suggested that molecules possessing these important scaffolds (piperazine/morpholine and dihydropyrimidinone) may have significant therapeutic activity. In the present disclosure, a series of novel piperazine/morpholine dihydropyrimidinone hybrids were prepared and analyzed by spectral data. 
<smiles>CC(C)(C)NCC(O)COc1nsnc1N1CCOCC1</smiles>

Timolol

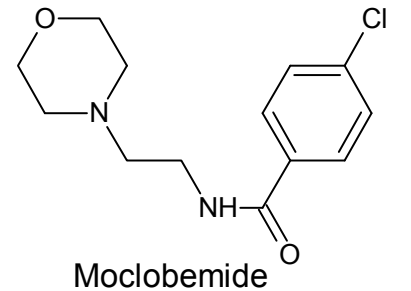

Moclobemide<smiles>CCOc1c(N2CCOCC2)cnn(C)c1=O</smiles>

Emorfazone

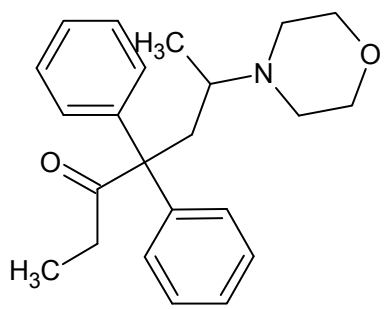

Phenadoxone<smiles>CCOc1ccccc1O[C@H](c1ccccc1)[C@H]1CNCCO1</smiles>

Reboxetine<smiles>COc1cc2ncnc(Nc3ccc(F)c(Cl)c3)c2cc1OCCCN1CCOCC1</smiles>

Gefitinib<smiles>CC(Cc1ccc(C(C)(C)C)cc1)CN1C[C@H](C)O[C@H](C)C1</smiles>

Fenpropimorph<smiles>CC1COc2c(N3CCN(C)CC3)c(F)cc3c(=O)c(C(=O)O)cn1c23</smiles>

Finafloxacin

Levofloxacin

Figure 2. Marketed drugs containing morpholine moiety.

\section{Results and Discussion}

As shown in (Scheme 1), enaminones, 4-methyl-1-[4-(piperazin/morpholin-1-yl) phenyl] pent-2-en-1-one (IIa-b) were synthesized by refluxing 1-[4-(piperazin/morpholin-1-yl) phenyl] ethan-1-one (Ia-b) with dimethylformamide dimethylacetal (DMF-DMA), without solvent for $10 \mathrm{~h}$. To prepare the final dihydropyrimidinone derivatives, a mixture of substituted benzaldehyde $(0.01 \mathrm{~mol})$ III, enaminones (IIa/IIb) $(0.01 \mathrm{~mol})$, urea $(0.01 \mathrm{~mol}) \mathbf{I V}$, and glacial acetic acid $(10 \mathrm{~mL})$ was heated on a heating mantle under refluxing condition for $3 \mathrm{~h}$. The precipitates of compounds (1-20) were collected by vacuum filtration. The product was washed several times with water, and recrystallized from glacial acetic acid and ethanol mixture. ${ }^{1} \mathrm{H}$ NMR spectrum of (IIa) displayed two singlets at $\delta \mathrm{H}$ $2.89,3.12 \mathrm{ppm}$ due to the $N, N$-dimethyl protons and two doublets at $\delta \mathrm{H} 5.80-5.82$ and 7.63-7.65 ppm $(\mathrm{d}, J=14 \mathrm{~Hz})$ due to the ethylenic protons, in addition to the two doublets at the region $\delta \mathrm{H} 7.0 \mathrm{ppm}$ $(2 \mathrm{H}, \mathrm{d}$, aromatic) and $\delta \mathrm{H} 7.82 \mathrm{ppm}(2 \mathrm{H}, \mathrm{d}$, aromatic). The protons of piperazine moiety appears at $\delta \mathrm{H}$ $3.40\left(4 \mathrm{H}\right.$, singlet) and $3.52\left(4 \mathrm{H}\right.$, singlet). ${ }^{1} \mathrm{H}$ NMR spectrum of $(\mathbf{I I b})$ displayed two singlets at $\delta \mathrm{H} 2.90$, $3.12 \mathrm{ppm}$ due to the $N, N$-dimethyl protons and two doublets at $\delta \mathrm{H} 5.80-5.82$ and 7.63-7.65 ppm (d, $J=14 \mathrm{~Hz})$ due to the ethylenic protons, in addition to the two doublets at the region $\delta \mathrm{H} 6.94 \mathrm{ppm}(2 \mathrm{H}$, $\mathrm{d}$, aromatic) and $\delta \mathrm{H} 7.82 \mathrm{ppm}(2 \mathrm{H}, \mathrm{d}$, aromatic). The protons of morpholine moiety appears at $\delta \mathrm{H} 3.21$ $(4 \mathrm{H}$, singlet) and $3.74(4 \mathrm{H}$, singlet). The three-dimensional structure of enaminone (IIb) was confirmed 
by single crystal X-ray. The coupling constant $(J=14 \mathrm{~Hz})$ for the ethylenic protons indicated that the enaminones existed in the E-configuration. Single crystal X-ray crystallography also confirmed the E-configuration of the enaminone [36].<smiles>[X]CCN(CC)c1ccc(C(C)=O)cc1</smiles>

(la): $X=\mathrm{NH}$

(lb): $X=0$

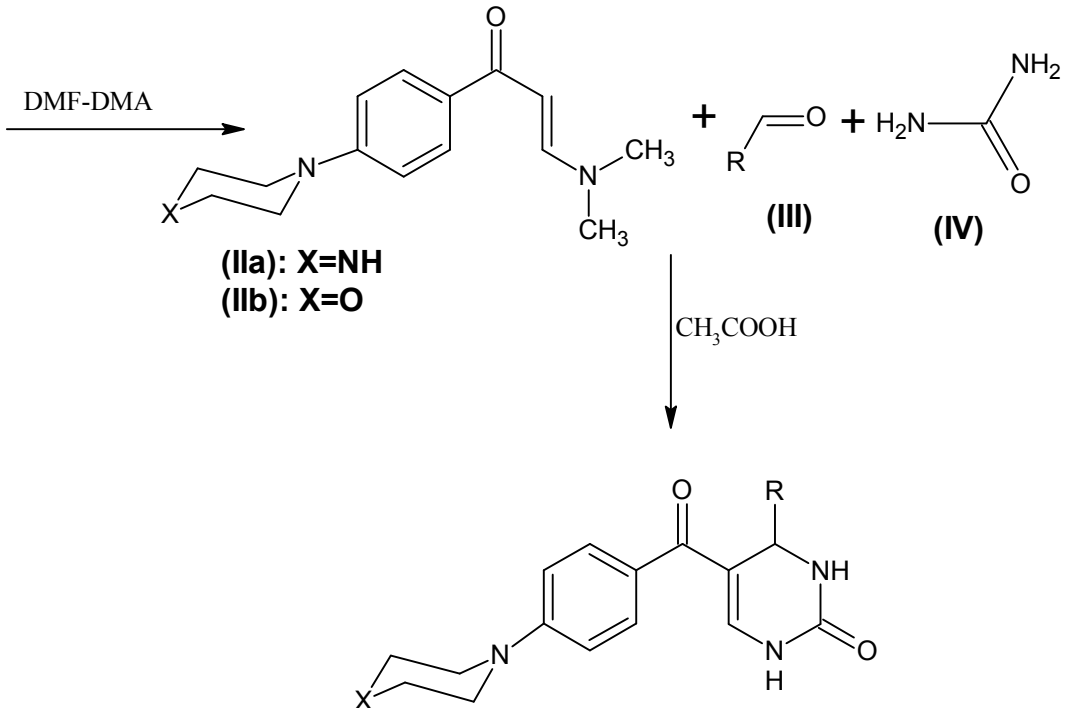

$(1-20)$

\begin{tabular}{|c|c|c|}
\hline Comp. & $\mathrm{X}$ & $\mathbf{R}$ \\
\hline 1 & $\mathrm{NH}$ & $\mathrm{C}_{6} \mathrm{H}_{5}-$ \\
\hline 2 & NH & $2-\mathrm{NO}_{2}-\mathrm{C}_{6} \mathrm{H}_{4}-$ \\
\hline 3 & $\mathrm{NH}$ & $4-\mathrm{NO}_{2}-\mathrm{C}_{6} \mathrm{H}_{4}-$ \\
\hline 4 & $\mathrm{NH}$ & $3-\mathrm{NO}_{2}-\mathrm{C}_{6} \mathrm{H}_{4}-$ \\
\hline 5 & $\mathrm{NH}$ & 4-Cl- $\mathrm{C}_{6} \mathrm{H}_{4-}-$ \\
\hline 6 & $\mathrm{NH}$ & $2-\mathrm{OCH}_{3}-\mathrm{C}_{6} \mathrm{H}_{4}-$ \\
\hline 7 & NH & $4-\mathrm{OH}-\mathrm{C}_{6} \mathrm{H}_{4-}^{-}$ \\
\hline 8 & $\mathrm{NH}$ & $3-\mathrm{OH}-\mathrm{C}_{6} \mathrm{H}_{4}-$ \\
\hline 9 & $\mathrm{NH}$ & $3-\mathrm{OCH}_{3}-\mathrm{C}_{6} \mathrm{H}_{4}-$ \\
\hline 10 & $\mathrm{NH}$ & $4-\mathrm{OC}_{2} \mathrm{H}_{5}-\mathrm{C}_{6} \mathrm{H}_{4}-$ \\
\hline 11 & $\mathrm{O}$ & $\mathrm{C}_{6} \mathrm{H}_{5-}$ \\
\hline 12 & $\mathrm{O}$ & 2- $\mathrm{NO}_{2}-\mathrm{C}_{6} \mathrm{H}_{4}^{-}$ \\
\hline 13 & $\mathrm{O}$ & $4-\mathrm{NO}_{2}-\mathrm{C}_{6} \mathrm{H}_{4}^{-}$ \\
\hline 14 & $\mathrm{O}$ & $3-\mathrm{NO}_{2}-\mathrm{C}_{6} \mathrm{H}_{4}-$ \\
\hline 15 & $\mathrm{O}$ & 4-Cl- $\mathrm{C}_{6} \mathrm{H}_{4-}-$ \\
\hline 16 & $\mathrm{O}$ & $2-\mathrm{OCH}_{3}-\mathrm{C}_{6} \mathrm{H}_{4}-$ \\
\hline 17 & $\mathrm{O}$ & $4-\mathrm{OH}-\mathrm{C}_{6} \mathrm{H}_{4}-$ \\
\hline 18 & $\mathrm{O}$ & $3-\mathrm{OH}-\mathrm{C}_{6} \mathrm{H}_{4}-$ \\
\hline 19 & $\mathrm{O}$ & $3-\mathrm{OCH}_{3}-\mathrm{C}_{6} \mathrm{H}_{4}-$ \\
\hline 20 & $\mathrm{O}$ & $4-\mathrm{OC}_{2} \mathrm{H}_{5}-\mathrm{C}_{6} \mathrm{H}_{4}-$ \\
\hline
\end{tabular}

Scheme 1. Reaction scheme for the synthesis of dihydropyrimidinone derivatives (1-20).

Compounds (1-20) presented the $\mathrm{D}_{2} \mathrm{O}$ exchangeable broad singlet at $\delta \mathrm{H} 6.71-8.52 \mathrm{ppm}$ and $\delta \mathrm{H}$ 9.00-9.42 ppm corresponding to the two NH protons. The eight protons $\left(4 \times \mathrm{CH}_{2}\right)$ of piperazine moiety

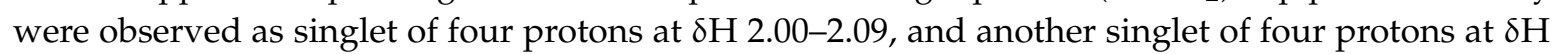
$3.20-3.41 \mathrm{ppm}$. The eight protons of morpholine moiety were observed as triplets at $\delta \mathrm{H} 3.20-3.22 \mathrm{ppm}$ with coupling constant $(J=4.7 \mathrm{~Hz})$ for four protons and another triplet at $\delta \mathrm{H} 3.72-3.82$ with coupling constant $(J=4.6 \mathrm{~Hz})$ for four protons. The $\mathrm{H}-4$ and $=\mathrm{CH}$ protons of dihydropyrimidinone moiety were observed at $\delta \mathrm{H} 5.32-6.08$ and 7.79-8.24 ppm, respectively [37-39]. ${ }^{13} \mathrm{C}$ NMR spectra confirmed the presence of all carbon atoms of compounds (1-20). 
Mass spectral data confirmed the molecular weight of compounds. All the compounds presented molecular ion peak respective to their molecular weights. The experimental part contains the detailed spectral results of ${ }^{1} \mathrm{H}$ NMR, ${ }^{13} \mathrm{C}$ NMR spectra, and mass spectra. The information regarding the crystallographic data and refinement of the compound (IIb), $\mathrm{C}_{15} \mathrm{H}_{20} \mathrm{~N}_{2} \mathrm{O}_{2}$ are summarized in Table 1 . The selected bond angles and bond lengths are listed in Table 2. Two independent molecules were found in the asymmetric unit as shown in Figure 3. All the bond lengths and angles were in normal ranges as reported [40]. The molecules were linked via two intermolecular hydrogen bonds in the crystal packing (Figure 4, Table 3).

Table 1. Experimental details.

\begin{tabular}{|c|c|}
\hline \multicolumn{2}{|c|}{ Crystal Data } \\
\hline Chemical formula & $\mathrm{C}_{15} \mathrm{H}_{20} \mathrm{~N}_{2} \mathrm{O}_{2}$ \\
\hline $\mathrm{Mr}$ & 260.33 \\
\hline Crystal system, space group & Triclinic, $P-1$ \\
\hline Temperature $(\mathrm{K})$ & 293 \\
\hline$a, b, c(\AA)$ & $9.5268(7), 10.2914(8), 15.3140(11)$ \\
\hline$\alpha \beta \gamma\left(^{\circ}\right)$ & $104.458(3), 97.224(3), 97.984(3)$ \\
\hline $\mathrm{V}(\AA 3)$ & $1419.51(18)$ \\
\hline Z & 4 \\
\hline Radiation type & Mo $K \alpha$ \\
\hline$\mu\left(\mathrm{mm}^{-1}\right)$ & 0.08 \\
\hline Crystal size $(\mathrm{mm})$ & $0.61 \times 0.31 \times 0.28$ \\
\hline \multicolumn{2}{|c|}{ Data collection } \\
\hline Diffractometer & Bruker APEX-II D8 venture diffractometer \\
\hline Absorption correction & Multi-scan SADABS Bruker 2014 \\
\hline Tmin, Tmax & $0.952,0.977$ \\
\hline $\begin{array}{l}\text { No. of measured, independent and observed } \\
\qquad[\mathrm{I}>2 \sigma(\mathrm{I})] \text { reflections }\end{array}$ & $27345,5007,2799$ \\
\hline $\mathrm{R}_{\text {int }}$ & 0.102 \\
\hline \multicolumn{2}{|c|}{ Refinement } \\
\hline $\mathrm{R}\left[F^{2}>2 \sigma\left(F^{2}\right)\right], \mathrm{wR}\left(F^{2}\right), \mathrm{S}$ & $0.075,0.239,1.04$ \\
\hline No. of reflections & 5007 \\
\hline No. of parameters & 348 \\
\hline No. of restraints & 0 \\
\hline $\mathrm{H}$-atom treatment & $\begin{array}{l}\mathrm{H} \text { atoms treated by a mixture of independent } \\
\text { and constrained refinement }\end{array}$ \\
\hline$\Delta \rho_{\max }, \Delta \rho_{\min }\left(\mathrm{e} \AA^{-3}\right)$ & $0.34,-0.32$ \\
\hline
\end{tabular}

Table 2. Selected geometric parameters (Å).

\begin{tabular}{cccc}
\hline O1A-C8A & $1.393(5)$ & N2A-C13A & $1.326(4)$ \\
O1A-C9A & $1.399(5)$ & N2A-C14A & $1.429(5)$ \\
O2A-C11A & $1.230(4)$ & N2A-C15A & $1.443(4)$ \\
O1B-C9B & $1.327(6)$ & N1B-C1B & $1.402(4)$ \\
O1B-C8B & $1.369(5)$ & N1B-C7B & $1.406(5)$ \\
O2B-C11B & $1.233(5)$ & N1B-C10B & $1.427(5)$ \\
N1A-C10A & $1.460(4)$ & N2B-C13B & $1.332(5)$ \\
N1A-C1A & $1.403(4)$ & N2B-C14B & $1.440(4)$ \\
N1A-C7A & $1.447(5)$ & N2B-C15B & $1.453(6)$ \\
C8A-O1A-C9A & $110.1(3)$ & N1A-C7A-C8A & $111.8(3)$ \\
C8B-O1B-C9B & $117.7(3)$ & O1A-C8A-C7A & $113.1(3)$ \\
C1A-N1A-C10A & $117.1(2)$ & O1A-C9A-C10A & $112.6(3)$ \\
C7A-N1A-C10A & $111.9(3)$ & N1A-C10A-C9A & $111.4(3)$ \\
C1A-N1A-C7A & $117.5(2)$ & O2A-C11A-C4A & $118.5(3)$ \\
\hline
\end{tabular}


Table 2. Cont

\begin{tabular}{cccc}
\hline C13A-N2A-C15A & $121.8(3)$ & O2A-C11A-C12A & $123.1(3)$ \\
C14A-N2A-C15A & $116.7(3)$ & N2A-C13A-C12A & $127.7(3)$ \\
C13A-N2A-C14A & $121.5(3)$ & N1B-C1B-C2B & $121.2(3)$ \\
C1B-N1B-C7B & $118.9(3)$ & N1B-C1B-C6B & $122.0(3)$ \\
C1B-N1B-C10B & $119.3(3)$ & N1B-C7B-C8B & $115.9(3)$ \\
C7B-N1B-C10B & $117.4(3)$ & O1B-C8B-C7B & $116.9(4)$ \\
C13B-N2B-C14B & $122.6(3)$ & O1B-C9B-C10B & $119.2(4)$ \\
C13B-N2B-C15B & $121.2(3)$ & N1B-C10B-C9B & $115.8(3)$ \\
C14B-N2B-C15B & $115.9(3)$ & O2B-C11B-C4B & $119.0(3)$ \\
N1A-C1A-C2A & $122.5(3)$ & O2B-C11B-C12B & $121.6(3)$ \\
N1A-C1A-C6A & $120.4(3)$ & N2B-C13B-C12B & $128.1(4)$ \\
\hline
\end{tabular}

Table 3. Hydrogen-bond geometry ( $)$.

\begin{tabular}{ccccc}
\hline $\mathbf{D}-\mathbf{H} \cdots \mathbf{A}$ & $\mathbf{D}-\mathbf{H}$ & $\mathbf{H} \cdots \mathbf{A}$ & $\mathbf{D} \cdots \mathbf{A}$ & $\mathbf{D}-\mathbf{H} \cdots \mathbf{A}$ \\
\hline $\mathrm{C} 5 \mathrm{~B}-\mathrm{H} 5 \mathrm{BA} \cdots \mathrm{O}^{2} \mathrm{~B}^{\mathrm{i}}$ & 0.930 & 2.5100 & $3.391(4)$ & 158.00 \\
$\mathrm{C} 13 \mathrm{~A}-\mathrm{H} 13 \mathrm{~A} \cdots \mathrm{O}^{2} \mathrm{~B}^{\mathrm{i}}$ & 0.930 & 2.5900 & $3.451(4)$ & 154.00 \\
$\mathrm{C} 13 \mathrm{~B}-\mathrm{H} 13 \mathrm{~B} \cdots \mathrm{O}^{\mathrm{i}} \mathrm{A}^{\mathrm{i}}$ & 0.930 & 2.5800 & $3.418(4)$ & 151.00 \\
$\mathrm{C} 15 \mathrm{~A}-\mathrm{H} 15 \mathrm{~A} \cdots \mathrm{O}^{\mathrm{B}} \mathrm{B}^{\mathrm{i}}$ & 0.960 & 2.5100 & $3.375(5)$ & 149.00 \\
& Symmetry code: $(\mathrm{i})-\mathrm{x}+1,-\mathrm{y}+1,-\mathrm{z}$. \\
\hline
\end{tabular}

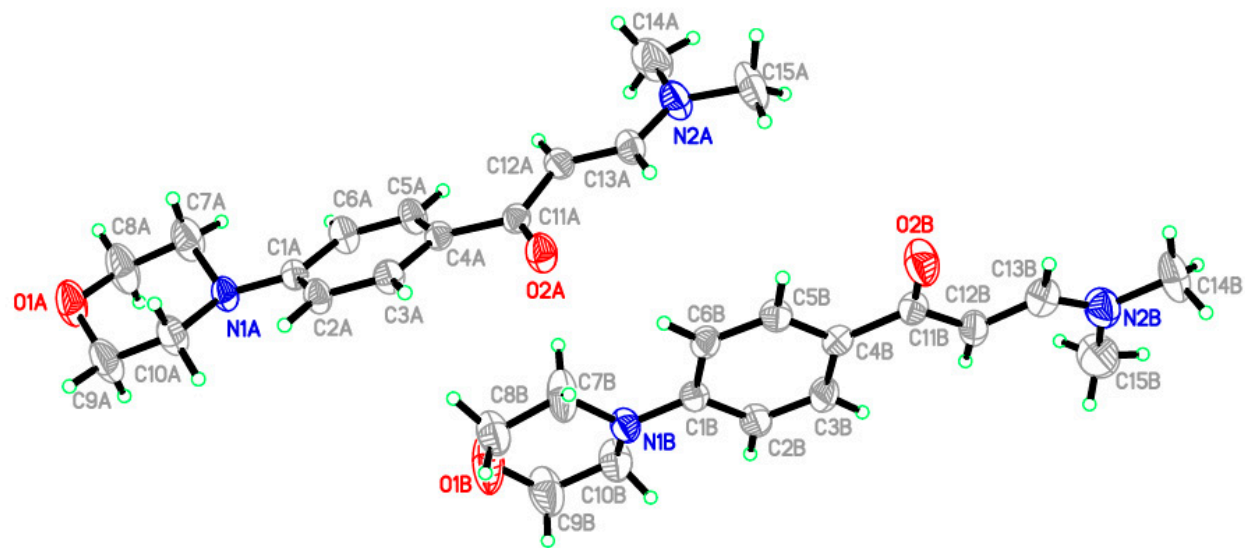

Figure 3. ORTEP diagram of the enaminone (IIb) containing morpholine moiety. Displacement ellipsoids are plotted at the $40 \%$ probability level for non-H atoms.

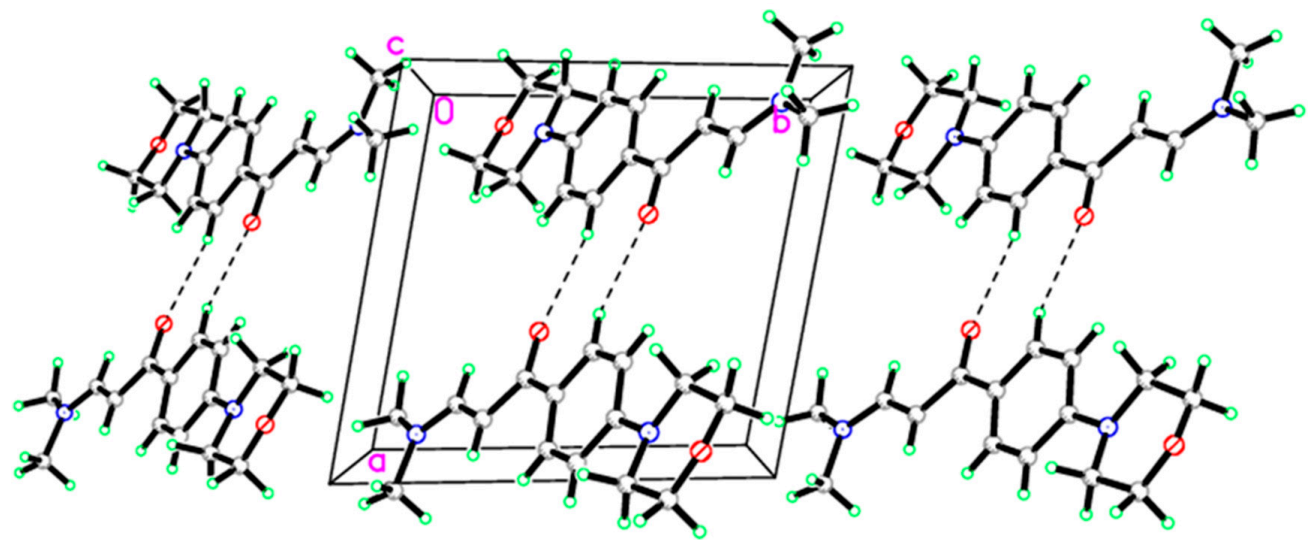

Figure 4. Molecular packing of enaminone (IIb) viewed hydrogen bonds which are drawn as dashed lines along $a$ axis. 
The mechanism involves the acid-catalyzed formation of iminium ion intermediate from the substituted benzaldehydes and urea. Reaction of iminium ion by enaminone of piperzine/morpholine produces ureidenone, which cyclizes to form hexahydropyrimidine. Elimination of $\mathrm{N}\left(\mathrm{CH}_{3}\right)_{2}$ group from hexahydropyrimidine in presence of glacial acetic acid produces final dihydropyrimidinone derivatives containing piperazine/morpholine moiety (Scheme 2).<smiles>[R]C(=O)[I+]C(N)=O</smiles><smiles>[R]C(O)NC(N)=O</smiles><smiles>[R]C=[NH+]C(N)=O</smiles><smiles>[X]CCN(CCC)c1ccc(C(=O)/C=C/N(C)C)cc1</smiles><smiles>[X]CCN(CC[X])c1ccc(C(=O)/C(=C/N(C)C)C([R])NC(N)=O)cc1</smiles>

(Ureidoenone)<smiles></smiles>

$(1-20)$<smiles>[X]CCN(CC)c1ccc(C(=O)C2C([R])NC(=O)NC2N(C)C)cc1</smiles>

(hexahydropyrimidinone)

Scheme 2. Mechanism of the reaction for the synthesis of dihydropyrimidinone derivatives (1-20).

\section{Material and Methods}

\subsection{Chemistry}

All the solvents were purchased from Merck (Kenilworth, NJ, USA). To check the purity of compounds, thin layer chromatography (TLC), was performed on silica gel $60 \mathrm{~F}_{254}$ coated plates (Merck). For performing FTIR, Perkin Elmer (Waltham, MA, USA) FT-IR spectrophotometer was used. Melting points were measured by Gallenkamp melting point apparatus. ${ }^{1} \mathrm{H}$ and ${ }^{13} \mathrm{C}$ NMR were recorded in Bruker (Billerica, MA, USA) NMR 500/700 MHz and 125/176 MHz spectrophotometer. The samples were run in DMSO- $d_{6}$ with tetramethyl silane (TMS) as an internal standard. Molecular 
weights of compounds were determined in mass spectroscopy. The elemental analysis of compounds was performed by CHN Elementar (Analysensysteme $\mathrm{GmbH}$, Langenselbold, Germany). The compound (IIb) was obtained as single crystal by reported method. Data were collected on a Bruker APEX-II D8 Venture area diffractometer. SHELXT was used to solve structure [41,42]. CCDC 1532829 contains the supplementary crystallographic data for the compound (IIb). These data can be obtained free of charge via http:/ / www.ccdc.cam.ac.uk/conts/retrieving.html (or from the CCDC, 12 Union Road, Cambridge CB2 1EZ, UK; Fax: +44 1223 336033; E-mail: deposit@ccdc.cam.ac.uk).

\subsection{Synthesis of 3-(dimethylamino)-1-(4-(piperazin-1-yl) phenyl)prop-2-en-1-one (IIa)}

A mixture of 1-[4-(piperazin-1-yl) phenyl]ethan-1-one (I) (0.02 mol) and dimethylformamide dimethylacetal (DMF-DMA) (II) $(0.023 \mathrm{~mol})$ was refluxed for $10 \mathrm{~h}$ without solvent, then, the reaction mixture was left to cool slowly at room temperature. Diethyl ether was added to reaction mixture. The precipitate was obtained and filtration was performed under vacuum. The product was washed with cold diethyl ether. The product so obtained was recrystallized from absolute ethanol. Yield: 92\%; m.p.: $105-107^{\circ} \mathrm{C}$; IR (KBr): vmax $/ \mathrm{cm}^{-1}$ : 1658 (C=O), 1541 (C=C), 1115 (C-O); ${ }^{1} \mathrm{H} \mathrm{NMR}(700 \mathrm{MHz}$, DMSO-d $\left.{ }_{6}\right) \delta$ ppm: $8.10(1 \mathrm{H}, \mathrm{s}, \mathrm{NH}), 7.82(2 \mathrm{H}, \mathrm{d}, J=14 \mathrm{~Hz}, \mathrm{Ar}-\mathrm{H}), 7.63-7.65(1 \mathrm{H}, \mathrm{d}, J=14 \mathrm{~Hz},=\mathrm{CH})$, $7.0(2 \mathrm{H}, \mathrm{d}, J=7 \mathrm{~Hz}, \mathrm{Ar}-\mathrm{H}) 5.80-5.82(1 \mathrm{H}, \mathrm{d}, J=14 \mathrm{~Hz},=\mathrm{CH}), 3.52(4 \mathrm{H}, \mathrm{s}$, piperazine $), 3.40(4 \mathrm{H}, \mathrm{s}$, piperazine), $3.12\left(3 \mathrm{H}, \mathrm{s}, \mathrm{N}-\mathrm{CH}_{3}\right), 2.89\left(3 \mathrm{H}, \mathrm{s}, \mathrm{N}-\mathrm{CH}_{3}\right) ;{ }^{13} \mathrm{C} \mathrm{NMR}\left(176.0 \mathrm{MHz}, \mathrm{DMSO}-d_{6}\right): \delta=26.2$, 44.6, 46.8, 48.0, 91.0, 114.1, 127.5, 129.2, 130.5, 153.7, 154.0, 161.4, 185.1, 196.1; MS: $m / z=259.16$ [M] $^{+}$; Analysis: for $\mathrm{C}_{15} \mathrm{H}_{21} \mathrm{~N}_{3} \mathrm{O}$, calcd. C 69.47, $\mathrm{H}$ 8.16, N 16.20\%; found C 69.20, H 8.14, N 16.14\%.

\subsection{Synthesis of 3-(dimethylamino)-1-(4-morpholinophenyl)prop-2-en-1-one (IIb)}

Yield: 90\%; m.p.: $210-212{ }^{\circ} \mathrm{C}$; IR (KBr): vmax $/ \mathrm{cm}^{-1}: 1640(\mathrm{C}=\mathrm{O}), 1540(\mathrm{C}=\mathrm{C}), 1111(\mathrm{C}-\mathrm{O}) ;{ }^{1} \mathrm{H}$ NMR (700 MHz, DMSO-d 6 ) $\delta$ ppm: $7.82(2 \mathrm{H}, \mathrm{d}, J=7 \mathrm{~Hz}, \mathrm{Ar}-\mathrm{H}), 7.63-7.65(1 \mathrm{H}, \mathrm{d}, J=14 \mathrm{~Hz},=\mathrm{CH})$, $6.94(2 \mathrm{H}, \mathrm{d}, J=7 \mathrm{~Hz}, \mathrm{Ar}-\mathrm{H}) 5.80-5.82(1 \mathrm{H}, \mathrm{d}, J=14 \mathrm{~Hz},=\mathrm{CH}), 3.74(4 \mathrm{H}, \mathrm{s}$, morpholine), 3.21 (4H, s, morpholine), $3.12\left(3 \mathrm{H}, \mathrm{s}, \mathrm{N}-\mathrm{CH}_{3}\right), 2.90\left(3 \mathrm{H}, \mathrm{s}, \mathrm{N}-\mathrm{CH}_{3}\right) ;{ }^{13} \mathrm{C} \mathrm{NMR}\left(176.0 \mathrm{MHz}, \mathrm{DMSO}-d_{6}\right): \delta=26.6,47.2$, 47.8, 66.42, 91.1, 113.7, 130.5, 130.7, 153.7, 185.1, 196.1; MS: $m / z=260.1[\mathrm{M}]^{+}$; Analysis: for $\mathrm{C}_{15} \mathrm{H}_{20} \mathrm{~N}_{2} \mathrm{O}_{2}$ ， calcd. C 69.20, H 7.74, N 10.76\%; found C 69.22, H 7.72, N 10.70\%.

\subsection{General Synthesis of 4-(substituted phenyl)-5-[4-(piperazin/morpholin-1-yl)benzoyl]-3,4-} dihydropyrimidin-2(1H)-one (1-20)

A mixture of enaminones, (2E)-4-methyl-1-[4-(piperazin/morpholin-1-yl) phenyl] pent-2-en-1-one $(0.01 \mathrm{~mol})$, differently substituted benzaldehydes $(0.01 \mathrm{~mol})$, urea $(0.01 \mathrm{~mol})$, and glacial acetic acid $(10 \mathrm{~mL})$ were refluxed for $3 \mathrm{~h}$ on a heating mantle. The reaction mixture was precipitated by pouring into the cold water. The products were obtained by vacuum filtration. The final products were recrystallized from glacial acetic acid and ethanol.

4-Phenyl-5-[4-(piperazin-1-yl)benzoyl]-3,4-dihydropyrimidin-2(1H)-one (1): Yield: 75\%; m.p.: $150-152{ }^{\circ} \mathrm{C}$; ${ }^{1} \mathrm{H}$ NMR (500 MHz, DMSO-d $): \delta=9.41\left(1 \mathrm{H}, \mathrm{s}, \mathrm{NH}, \mathrm{D}_{2} \mathrm{O}\right.$ exch), $8.50\left(1 \mathrm{H}, \mathrm{s}, \mathrm{NH}, \mathrm{D}_{2} \mathrm{O}\right.$ exch), 8.0 $(1 \mathrm{H}, \mathrm{s},=\mathrm{CH}), 6.90-7.80(9 \mathrm{H}, \mathrm{m}, \mathrm{Ar}-\mathrm{H}), 6.0(1 \mathrm{H}, \mathrm{s}, \mathrm{H}-4), 3.40\left(2 \mathrm{H}, \mathrm{s}, \mathrm{CH}_{2}\right.$ piperazine $), 3.31\left(2 \mathrm{H}, \mathrm{s}, \mathrm{CH}_{2}\right.$ piperazine), $2.06\left(2 \mathrm{H}, \mathrm{s}, \mathrm{CH}_{2}\right.$ piperazine $), 2.0\left(2 \mathrm{H}, \mathrm{s}, \mathrm{CH}_{2}\right.$ piperazine $), 1.80\left(1 \mathrm{H}, \mathrm{s}, \mathrm{NH}, \mathrm{D}_{2} \mathrm{O}\right.$ exch); ${ }^{13} \mathrm{C}$ NMR (125.76 MHz, DMSO-d $\left.d_{6}\right): \delta=44.6\left(\mathrm{CH}_{2}\right), 47.0\left(\mathrm{CH}_{2}\right), 48.0(\mathrm{CH}), 50.10\left(\mathrm{CH}_{2}\right), 65.5\left(\mathrm{CH}_{2}\right), 111.5$, 113.0, 114.0, 114.2, 124.4, 130.5, 134.1, 138.7, 148.0, 149.0, 151.0, 161.1, 168.0, $190.40(\mathrm{C}=\mathrm{O}), 207.0(\mathrm{C}=\mathrm{O})$ MS: $m / z=362.42[\mathrm{M}]^{+}$; Analysis: for $\mathrm{C}_{21} \mathrm{H}_{22} \mathrm{~N}_{4} \mathrm{O}_{2}$, calcd. C 69.59, $\mathrm{H} 6.12, \mathrm{~N} 15.46 \%$; found C 69.32, H 6.10, N $15.40 \%$.

4-(2-Nitrophenyl)-5-[4-(piperazin-1-yl)benzoyl]-3,4-dihydropyrimidin-2(1H)-one (2): Yield: 70\%; m.p.: 170-172 ${ }^{\circ} \mathrm{C} ;{ }^{1} \mathrm{H}$ NMR (500 MHz, DMSO-d 6 ): $\delta=9.40\left(1 \mathrm{H}, \mathrm{s}, \mathrm{NH}, \mathrm{D}_{2} \mathrm{O}\right.$ exch), $8.52\left(1 \mathrm{H}, \mathrm{s}, \mathrm{NH}, \mathrm{D}_{2} \mathrm{O}\right.$ exch), $8.04(1 \mathrm{H}, \mathrm{s},=\mathrm{CH}), 6.89-7.88(8 \mathrm{H}, \mathrm{m}, \mathrm{Ar}-\mathrm{H}), 6.07(1 \mathrm{H}, \mathrm{s}, \mathrm{H}-4), 3.41\left(2 \mathrm{H}, \mathrm{s}, \mathrm{CH}_{2}\right.$ piperazine $), 3.32(2 \mathrm{H}, \mathrm{s}$, $\mathrm{CH}_{2}$ piperazine), $2.06\left(2 \mathrm{H}, \mathrm{s}, \mathrm{CH}_{2}\right.$ piperazine $), 2.0\left(2 \mathrm{H}, \mathrm{s}, \mathrm{CH}_{2}\right.$ piperazine $), 1.79\left(1 \mathrm{H}, \mathrm{s}, \mathrm{NH}, \mathrm{D}_{2} \mathrm{O}\right.$ exch $) ;{ }^{13} \mathrm{C}$ NMR (125.76 MHz, DMSO-d $\left.)_{6}\right): \delta=44.7\left(\mathrm{CH}_{2}\right), 47.2\left(\mathrm{CH}_{2}\right), 48.4(\mathrm{CH}), 50.11\left(\mathrm{CH}_{2}\right), 65.4\left(\mathrm{CH}_{2}\right), 111.7$, 
113.6, 114.1, 114.5, 124.4, 130.5, 134.1, 138.7, 148.3, 149.1, 151.2, 161.5, 168.9, 190.40 (C=O), 207.0 (C=O); MS: $m / z=407.40[\mathrm{M}]^{+}$; Analysis: for $\mathrm{C}_{21} \mathrm{H}_{21} \mathrm{~N}_{5} \mathrm{O}_{4}$, calcd. C 61.91, H 5.20, N 17.19\%; found C 62.15, $\mathrm{H}$ $5.22, \mathrm{~N} 17.12 \%$.

4-(4-Nitrophenyl)-5-[4-(piperazin-1-yl)benzoyl]-3,4-dihydropyrimidin-2(1H)-one (3): Yield: 75\%; m.p.: 175-177 ${ }^{\circ} \mathrm{C} ;{ }^{1} \mathrm{H}$ NMR (500 MHz, DMSO-d 6 ): $\delta=9.41\left(1 \mathrm{H}, \mathrm{s}, \mathrm{NH}, \mathrm{D}_{2} \mathrm{O}\right.$ exch), $8.28(1 \mathrm{H}, \mathrm{s},=\mathrm{CH})$, 6.93-7.58 (8H, m, Ar-H), $7.93\left(1 \mathrm{H}, \mathrm{s}, \mathrm{NH}, \mathrm{D}_{2} \mathrm{O}\right.$ exch), $5.55(1 \mathrm{H}, \mathrm{s}, \mathrm{H}-4), 3.30$ (2H, s, $\mathrm{CH}_{2}$ piperazine), $3.23\left(2 \mathrm{H}, \mathrm{s}, \mathrm{CH}_{2}\right.$ piperazine), $2.07\left(2 \mathrm{H}, \mathrm{s}, \mathrm{CH}_{2}\right.$ piperazine $), 2.0\left(2 \mathrm{H}, \mathrm{s}, \mathrm{CH}_{2}\right.$ piperazine $), 1.85(1 \mathrm{H}, \mathrm{s}, \mathrm{NH}$, $\mathrm{D}_{2} \mathrm{O}$ exch); ${ }^{13} \mathrm{C}$ NMR $\left(125.76 \mathrm{MHz}, \mathrm{DMSO}-d_{6}\right): \delta=44.7\left(\mathrm{CH}_{2}\right), 45.5\left(\mathrm{CH}_{2}\right), 47.5(\mathrm{CH}), 48.0\left(\mathrm{CH}_{2}\right), 53.9$ $\left(\mathrm{CH}_{2}\right), 118.8,114.1,124.3,128.3,130.6,147.2,151.6,153.1,161.3,168.8,190.4(\mathrm{C}=\mathrm{O}), 207.0(\mathrm{C}=\mathrm{O})$; $\mathrm{MS}$ : $m / z=407.42[\mathrm{M}]^{+}$; Analysis: for $\mathrm{C}_{21} \mathrm{H}_{21} \mathrm{~N}_{5} \mathrm{O}_{4}$, calcd. C 61.91, H 5.20, N 17.19\%; found C 62.10, H 5.23, $\mathrm{N} 17.13 \%$.

4-(3-Nitrophenyl)-5-[4-(piperazin-1-yl)benzoyl]-3,4-dihydropyrimidin-2(1H)-one (4): Yield: 75\%; m.p.: 180-182 ${ }^{\circ} \mathrm{C} ;{ }^{1} \mathrm{H}$ NMR (500 MHz, DMSO-d $): \delta=9.42\left(1 \mathrm{H}, \mathrm{s}, \mathrm{NH}, \mathrm{D}_{2} \mathrm{O}\right.$ exch), $8.23(1 \mathrm{H}, \mathrm{s},=\mathrm{CH})$, 6.93-7.65 (8H, m, Ar-H), $7.95\left(1 \mathrm{H}, \mathrm{s}, \mathrm{NH}, \mathrm{D}_{2} \mathrm{O}\right.$ exch), 5.58 (1H, s, H-4), 3.31 (2H, s, $\mathrm{CH}_{2}$ piperazine), 3.23 ( $2 \mathrm{H}, \mathrm{s}, \mathrm{CH}_{2}$ piperazine), $2.07\left(2 \mathrm{H}, \mathrm{s}, \mathrm{CH}_{2}\right.$ piperazine $), 2.01\left(2 \mathrm{H}, \mathrm{s}, \mathrm{CH}_{2}\right.$ piperazine $), 1.87\left(1 \mathrm{H}, \mathrm{s}, \mathrm{NH}, \mathrm{D}_{2} \mathrm{O}\right.$ exch); ${ }^{13} \mathrm{C}$ NMR (125.76 MHz, DMSO-d $): \delta=44.7\left(\mathrm{CH}_{2}\right), 47.2\left(\mathrm{CH}_{2}\right), 48.0(\mathrm{CH}), 53.8\left(\mathrm{CH}_{2}\right), 65.4\left(\mathrm{CH}_{2}\right)$, 111.8, 114.1, 121.6, 122.9, 128.3, 130.3, 131.3, 133.7, 146.7, 148.2, 151.6, 161.3, 168.8, 190.5 (C=O), 207.0 $(\mathrm{C}=\mathrm{O})$; MS: $m / z=407.42[\mathrm{M}]^{+}$; Analysis: for $\mathrm{C}_{21} \mathrm{H}_{21} \mathrm{~N}_{5} \mathrm{O}_{4}$, calcd. C 61.91, H 5.20, N 17.19\%; found C 60.67, H 5.22, N $17.10 \%$.

4-(4-Chlorophenyl)-5-[4-(piperazin-1-yl)benzoyl]-3,4-dihydropyrimidin-2(1H)-one (5): Yield: 75\%; m.p.: 160-162 ${ }^{\circ} \mathrm{C} ;{ }^{1} \mathrm{H}$ NMR (500 MHz, DMSO-d $): \delta=9.32\left(1 \mathrm{H}, \mathrm{s}, \mathrm{NH}, \mathrm{D}_{2} \mathrm{O}\right.$ exch), $8.50\left(1 \mathrm{H}, \mathrm{s}, \mathrm{NH}, \mathrm{D}_{2} \mathrm{O}\right.$ exch), $8.23(1 \mathrm{H}, \mathrm{s},=\mathrm{CH}), 6.95-8.11(8 \mathrm{H}, \mathrm{m}, \mathrm{Ar}-\mathrm{H}), 5.46(1 \mathrm{H}, \mathrm{s}, \mathrm{H}-4), 3.31\left(2 \mathrm{H}, \mathrm{s}, \mathrm{CH}_{2}\right.$ piperazine $), 3.23$ (2H, s, $\mathrm{CH}_{2}$ piperazine), $2.09\left(2 \mathrm{H}, \mathrm{s}, \mathrm{CH}_{2}\right.$ piperazine $), 2.04\left(2 \mathrm{H}, \mathrm{s}, \mathrm{CH}_{2}\right.$ piperazine $), 1.90\left(1 \mathrm{H}, \mathrm{s}, \mathrm{NH}, \mathrm{D}_{2} \mathrm{O}\right.$ exch); ${ }^{13} \mathrm{C}$ NMR (125.76 MHz, DMSO-d 6$): \delta=44.7\left(\mathrm{CH}_{2}\right), 47.3\left(\mathrm{CH}_{2}\right), 48.4(\mathrm{CH}), 53.6\left(\mathrm{CH}_{2}\right), 65.4\left(\mathrm{CH}_{2}\right)$, 112.5, 114.1, 115.6, 116.0, 128.5, 140.0, 143.6, 148.1, 151.8, 153.1, 156.7, 161.3, 168.7, 190.5 (C=O), 207.0 $(\mathrm{C}=\mathrm{O})$; MS: $m / z=396.87[\mathrm{M}]^{+}$; Analysis: for $\mathrm{C}_{21} \mathrm{H}_{21} \mathrm{ClN}_{4} \mathrm{O}_{2}$, calcd. C 63.55, H 5.33, N 14.12\%; found C 63.31, H 5.34, N 14.17\%.

4-(2-Methoxyphenyl)-5-[4-(piperazin-1-yl)benzoyl]-3,4-dihydropyrimidin-2(1H)-one (6): Yield: 75\%; m.p.: 120-122 ${ }^{\circ} \mathrm{C} ;{ }^{1} \mathrm{H}$ NMR (500 MHz, DMSO-d $): \delta=9.22\left(1 \mathrm{H}, \mathrm{s}, \mathrm{NH}, \mathrm{D}_{2} \mathrm{O}\right.$ exch), $8.51\left(1 \mathrm{H}, \mathrm{s}, \mathrm{NH}, \mathrm{D}_{2} \mathrm{O}\right.$ exch), $8.13(1 \mathrm{H}, \mathrm{s},=\mathrm{CH}), 6.93-7.98(8 \mathrm{H}, \mathrm{m}, \mathrm{Ar}-\mathrm{H}), 5.77(1 \mathrm{H}, \mathrm{s}, \mathrm{H}-4), 3.82\left(3 \mathrm{H}, \mathrm{s}, \mathrm{OCH}_{3}\right), 3.41\left(2 \mathrm{H}, \mathrm{s}, \mathrm{CH}_{2}\right.$ piperazine), 3.24 (2H, s, $\mathrm{CH}_{2}$ piperazine), 2.09 ( $2 \mathrm{H}, \mathrm{s}, \mathrm{CH}_{2}$ piperazine), $2.04\left(2 \mathrm{H}, \mathrm{s}, \mathrm{CH}_{2}\right.$ piperazine), 1.93 $\left(1 \mathrm{H}, \mathrm{s}, \mathrm{NH}, \mathrm{D}_{2} \mathrm{O}\right.$ exch); ${ }^{13} \mathrm{C}$ NMR $\left(125.76 \mathrm{MHz}, \mathrm{DMSO}-d_{6}\right): \delta=44.6\left(\mathrm{CH}_{2}\right), 46.7\left(\mathrm{CH}_{2}\right), 49.6(\mathrm{CH}), 55.9$ $\left(\mathrm{CH}_{2}\right), 56.1\left(\mathrm{CH}_{2}\right), 111.1,114.2,120.6,130.5,131.0,137.3,154.0,157.3,158.5,161.4,168.8,172.6,187.1$, 190.5, $196.1(\mathrm{C}=\mathrm{O}), 207.0(\mathrm{C}=\mathrm{O})$; MS: $m / z=392.45[\mathrm{M}]^{+}$; Analysis: for $\mathrm{C}_{22} \mathrm{H}_{24} \mathrm{~N}_{4} \mathrm{O}_{3}$, calcd. C 67.33, $\mathrm{H}$ 6.16, N 14.28\%; found C 67.58, H 6.14, N 14.23\%.

4-(4-Hydroxyphenyl)-5-[4-(piperazin-1-yl)benzoyl]-3,4-dihydropyrimidin-2(1H)-one (7): Yield: 75\%; m.p.: 210-212 ${ }^{\circ} \mathrm{C} ;{ }^{1} \mathrm{H}$ NMR (500 MHz, DMSO-d $): \delta=9.16\left(1 \mathrm{H}, \mathrm{s}, \mathrm{OH}, \mathrm{D}_{2} \mathrm{O}\right.$ exch), $9.0\left(1 \mathrm{H}, \mathrm{s}, \mathrm{NH}, \mathrm{D}_{2} \mathrm{O}\right.$ exch), $8.51\left(1 \mathrm{H}, \mathrm{s}, \mathrm{NH}, \mathrm{D}_{2} \mathrm{O}\right.$ exch), $8.17(1 \mathrm{H}, \mathrm{s},=\mathrm{CH}), 6.71-8.08$ (8H, m, Ar-H), 5.33 (1H, s, H-4), 3.35 (2H, s, $\mathrm{CH}_{2}$ piperazine), $3.20\left(2 \mathrm{H}, \mathrm{s}, \mathrm{CH}_{2}\right.$ piperazine $), 2.07\left(2 \mathrm{H}, \mathrm{s}, \mathrm{CH}_{2}\right.$ piperazine $), 2.01\left(2 \mathrm{H}, \mathrm{s}, \mathrm{CH}_{2}\right.$ piperazine $)$, $1.82\left(1 \mathrm{H}, \mathrm{s}, \mathrm{NH}, \mathrm{D}_{2} \mathrm{O}\right.$ exch); ${ }^{13} \mathrm{C} \mathrm{NMR}\left(125.76 \mathrm{MHz}, \mathrm{DMSO}-d_{6}\right): \delta=44.7\left(\mathrm{CH}_{2}\right), 48.1\left(\mathrm{CH}_{2}\right), 49.2(\mathrm{CH})$, $53.5\left(\mathrm{CH}_{2}\right), 65.4\left(\mathrm{CH}_{2}\right), 115.4,116.0,128.0,129.6,130.3,151.7,152.0,153.0,155.8,156.4,157.1,159.1$, 161.3, 168.8, $190.7(\mathrm{C}=\mathrm{O}), 207.0(\mathrm{C}=\mathrm{O})$; MS: $m / z=378.42[\mathrm{M}]^{+}$; Analysis: for $\mathrm{C}_{21} \mathrm{H}_{22} \mathrm{~N}_{4} \mathrm{O}_{3}$, calcd. C 66.65, H 5.86, N 14.81\%; found C 66.40, H 5.84, N 14.86\%.

4-(3-Hydroxyphenyl)-5-[4-(piperazin-1-yl)benzoyl]-3,4-dihydropyrimidin-2(1H)-one (8): Yield: 75\%; m.p.: 158-160 ${ }^{\circ} \mathrm{C} ;{ }^{1} \mathrm{H}$ NMR (500 MHz, DMSO-d $): \delta=9.8\left(1 \mathrm{H}, \mathrm{s}, \mathrm{OH}, \mathrm{D}_{2} \mathrm{O}\right.$ exch), $9.10\left(1 \mathrm{H}, \mathrm{s}, \mathrm{NH}, \mathrm{D}_{2} \mathrm{O}\right.$ exch), $8.04(1 \mathrm{H}, \mathrm{s},=\mathrm{CH}), 6.82-7.77(8 \mathrm{H}, \mathrm{m}, \mathrm{Ar}-\mathrm{H}), 6.71\left(1 \mathrm{H}, \mathrm{s}, \mathrm{NH}, \mathrm{D}_{2} \mathrm{O}\right.$ exch), $5.32(1 \mathrm{H}, \mathrm{s}, \mathrm{H}-4), 3.30$ (2H, s, $\mathrm{CH}_{2}$ piperazine), $3.20\left(2 \mathrm{H}, \mathrm{s}, \mathrm{CH}_{2}\right.$ piperazine $), 2.04\left(2 \mathrm{H}, \mathrm{s}, \mathrm{CH}_{2}\right.$ piperazine $), 2.0\left(2 \mathrm{H}, \mathrm{s}, \mathrm{CH}_{2}\right.$ piperazine $), 1.86$ $\left(1 \mathrm{H}, \mathrm{s}, \mathrm{NH}, \mathrm{D}_{2} \mathrm{O}\right.$ exch); ${ }^{13} \mathrm{C}$ NMR (125.76 MHz, DMSO-d 6$): \delta=44.6\left(\mathrm{CH}_{2}\right), 46.7\left(\mathrm{CH}_{2}\right), 48.0(\mathrm{CH}), 53.8$ 
$\left(\mathrm{CH}_{2}\right), 56.5\left(\mathrm{CH}_{2}\right), 115.5,117.3,120.1,122.9,128.6,130.5,136.7,139.5,143.0,146.1,152.1,153.1,154.0$, 157.8, 161.4, 168.8, 172.6, 187.0, 190.6, 196.6 (C=O), $207.0(\mathrm{C}=\mathrm{O})$; MS: $m / z=378.42[\mathrm{M}]^{+}$; Analysis: for $\mathrm{C}_{21} \mathrm{H}_{22} \mathrm{~N}_{4} \mathrm{O}_{3}$, calcd. C 66.65, H 5.86, N 14.81\%; found C 66.39, H 5.83, N 14.85\%.

4-(3-Methoxyphenyl)-5-[4-(piperazin-1-yl)benzoyl]-3,4-dihydropyrimidin-2(1H)-one (9): Yield: 70\%; m.p.: 118-120 ${ }^{\circ} \mathrm{C} ;{ }^{1} \mathrm{H}$ NMR $\left(500 \mathrm{MHz}\right.$, DMSO- $\left.d_{6}\right): \delta=9.28\left(1 \mathrm{H}, \mathrm{s}, \mathrm{NH}, \mathrm{D}_{2} \mathrm{O}\right.$ exch), $8.50\left(1 \mathrm{H}, \mathrm{s}, \mathrm{NH}, \mathrm{D}_{2} \mathrm{O}\right.$ exch), $8.24(1 \mathrm{H}, \mathrm{s},=\mathrm{CH}), 6.88-8.11(8 \mathrm{H}, \mathrm{m}, \mathrm{Ar}-\mathrm{H}), 5.47(1 \mathrm{H}, \mathrm{s}, \mathrm{H}-4), 3.73\left(3 \mathrm{H}, \mathrm{s}, \mathrm{OCH}_{3}\right), 3.33(2 \mathrm{H}, \mathrm{s}$, $\mathrm{CH}_{2}$ piperazine), $3.20\left(2 \mathrm{H}, \mathrm{s}, \mathrm{CH}_{2}\right.$ piperazine $), 2.09\left(2 \mathrm{H}, \mathrm{s}, \mathrm{CH}_{2}\right.$ piperazine $), 2.04\left(2 \mathrm{H}, \mathrm{s}, \mathrm{CH}_{2}\right.$ piperazine $)$, $1.92\left(1 \mathrm{H}, \mathrm{s}, \mathrm{NH}, \mathrm{D}_{2} \mathrm{O}\right.$ exch); ${ }^{13} \mathrm{C}$ NMR $\left(125.76 \mathrm{MHz}, \mathrm{DMSO}-d_{6}\right): \delta=44.6\left(\mathrm{CH}_{2}\right), 46.8\left(\mathrm{CH}_{2}\right), 47.3(\mathrm{CH})$, $55.4\left(\mathrm{CH}_{2}\right), 65.4\left(\mathrm{CH}_{2}\right), 112.7,114.1,118.9,130.5,136.8,139.9,142.8,146.1,151.7,152.0,153.1,154.0$, 159.7, 160.1, 161.4 172.7, 187.0, 190.6, $196.1(\mathrm{C}=\mathrm{O}), 207.0(\mathrm{C}=\mathrm{O})$; MS: $m / z=392.45$ [M] ${ }^{+}$; Analysis: for $\mathrm{C}_{22} \mathrm{H}_{24} \mathrm{~N}_{4} \mathrm{O}_{3}$, calcd. C 67.33, H 6.16, N 14.28\%; found C 67.57, H 6.14, N 14.23\%.

4-(3-Ethoxyphenyl)-5-[4-(piperazin-1-yl)benzoyl]-3,4-dihydropyrimidin-2(1H)-one (10): Yield: 65\%; m.p.: $88-90{ }^{\circ} \mathrm{C} ;{ }^{1} \mathrm{H}$ NMR $\left(500 \mathrm{MHz}, \mathrm{DMSO}-d_{6}\right): \delta=9.21\left(1 \mathrm{H}, \mathrm{s}, \mathrm{NH}, \mathrm{D}_{2} \mathrm{O}\right.$ exch), $8.52\left(1 \mathrm{H}, \mathrm{s}, \mathrm{NH}, \mathrm{D}_{2} \mathrm{O}\right.$ exch), $8.20(1 \mathrm{H}, \mathrm{s},=\mathrm{CH}), 6.87-8.11(8 \mathrm{H}, \mathrm{m}, \mathrm{Ar}-\mathrm{H}), 5.40(1 \mathrm{H}, \mathrm{s}, \mathrm{H}-4), 4.0\left(2 \mathrm{H}, \mathrm{q}, \mathrm{OCH}_{2}\right), 3.33\left(2 \mathrm{H}, \mathrm{s}, \mathrm{CH}_{2}\right.$ piperazine), $3.20\left(2 \mathrm{H}, \mathrm{s}, \mathrm{CH}_{2}\right.$ piperazine $), 2.09\left(2 \mathrm{H}, \mathrm{s}, \mathrm{CH}_{2}\right.$ piperazine $), 2.08\left(2 \mathrm{H}, \mathrm{s}, \mathrm{CH}_{2}\right.$ piperazine $), 1.92$ $\left(1 \mathrm{H}, \mathrm{s}, \mathrm{NH}, \mathrm{D}_{2} \mathrm{O}\right.$ exch), $1.35\left(3 \mathrm{H}, \mathrm{t}, \mathrm{CH}_{3}\right) ;{ }^{13} \mathrm{C} \mathrm{NMR}\left(125.76 \mathrm{MHz}, \mathrm{DMSO}-d_{6}\right): \delta=15.0\left(\mathrm{CH}_{3}\right), 44.6$ $\left(\mathrm{OCH}_{2}\right), 46.8\left(\mathrm{CH}_{2}\right), 47.3\left(\mathrm{CH}_{2}\right), 48.0(\mathrm{CH}), 48.5\left(\mathrm{CH}_{2}\right), 63.7\left(\mathrm{CH}_{2}\right), 114.2,115.1,128.0,130.5,130.9,131.0$, $153.9,158.2,160.8,161.4,172.5,186.9,190.6,191.7,196.1(\mathrm{C}=\mathrm{O}), 207.0(\mathrm{C}=\mathrm{O}) ; \mathrm{MS}: m / z=406.43[\mathrm{M}]^{+}$; Analysis: for $\mathrm{C}_{23} \mathrm{H}_{26} \mathrm{~N}_{4} \mathrm{O}_{3}$, calcd. C 67.96, H 6.45, N 13.78\%; found C 67.70, H 4.46, N 13.73\%.

5-[4-(Morpholin-4-yl)benzoyl]-4-phenyl-3,4-dihydropyrimidin-2(1H)-one (11): Yield: 70\%; m.p.: 258-260 ${ }^{\circ} \mathrm{C}$; ${ }^{1} \mathrm{H}$ NMR $\left(500 \mathrm{MHz}, \mathrm{DMSO}-d_{6}\right): \delta=9.21\left(1 \mathrm{H}, \mathrm{s}, \mathrm{NH}, \mathrm{D}_{2} \mathrm{O}\right.$ exch), $7.79(1 \mathrm{H}, \mathrm{s},=\mathrm{CH}), 7.09-7.45(6 \mathrm{H}, \mathrm{m}$, $\mathrm{Ar}-\mathrm{H}), 7.01\left(1 \mathrm{H}, \mathrm{s}, \mathrm{NH}, \mathrm{D}_{2} \mathrm{O}\right.$ exch), $6.95(3 \mathrm{H}, \mathrm{m}, \mathrm{Ar}-\mathrm{H}), 5.44(1 \mathrm{H}, \mathrm{s}, \mathrm{H}-4), 3.74\left(4 \mathrm{H}, \mathrm{t}, J=4.6 \mathrm{~Hz}, 2 \times \mathrm{CH}_{2}\right.$ morpholine), $3.22\left(4 \mathrm{H}, \mathrm{t}, J=4.8 \mathrm{~Hz}, 2 \times \mathrm{CH}_{2}\right.$ morpholine $) ;{ }^{13} \mathrm{C} \mathrm{NMR}\left(125.76 \mathrm{MHz}, \mathrm{DMSO}-d_{6}\right): \delta=47.6$ $\left(\mathrm{CH}_{2}\right), 47.7\left(\mathrm{CH}_{2}\right), 54.0(\mathrm{CH}), 66.35\left(\mathrm{CH}_{2}\right), 66.37\left(\mathrm{CH}_{2}\right), 112.9,113.81,113.84,126.8,127.8,128.5,128.9$, 130.4, 130.5, 139.8, 144.6, 151.9, 153.3, 153.5, $190.6(\mathrm{C}=\mathrm{O}), 194.0(\mathrm{C}=\mathrm{O})$; MS: $m / z=363.42[\mathrm{M}]^{+}$; Analysis: for $\mathrm{C}_{21} \mathrm{H}_{21} \mathrm{~N}_{3} \mathrm{O}_{3}$, calcd. C 69.41, H 5.82, N 11.56\%; found C 69.58, H 5.80, N 11.59\%.

5-[4-(Morpholin-4-yl)benzoyl]-4-(2-nitrophenyl)-3,4-dihydropyrimidin-2(1H)-one (12): Yield: 75\%; m.p.: 198-200 ${ }^{\circ} \mathrm{C} ;{ }^{1} \mathrm{H}$ NMR $\left(500 \mathrm{MHz}, \mathrm{DMSO}-d_{6}\right): \delta=9.42\left(1 \mathrm{H}, \mathrm{d}, \mathrm{NH}, \mathrm{D}_{2} \mathrm{O}\right.$ exch), $8.07(1 \mathrm{H}, \mathrm{s},=\mathrm{CH})$, 7.06-7.91 (8H, m, Ar-H), $6.93\left(1 \mathrm{H}, \mathrm{s}, \mathrm{NH}, \mathrm{D}_{2} \mathrm{O}\right.$ exch), $6.08(1 \mathrm{H}, \mathrm{s}, \mathrm{H}-4), 3.73\left(4 \mathrm{H}, \mathrm{t}, J=4.6 \mathrm{~Hz}, 2 \times \mathrm{CH}_{2}\right.$ morpholine), $3.21\left(4 \mathrm{H}, \mathrm{t}, J=4.7 \mathrm{~Hz}, 2 \times \mathrm{CH}_{2}\right.$ morpholine $) ;{ }^{13} \mathrm{C} \mathrm{NMR}\left(125.76 \mathrm{MHz}, \mathrm{DMSO}-d_{6}\right): \delta=47.0$ $\left(\mathrm{CH}_{2}\right), 47.6\left(\mathrm{CH}_{2}\right), 50.0(\mathrm{CH}), 66.2\left(\mathrm{CH}_{2}\right), 66.3\left(\mathrm{CH}_{2}\right), 111.7,113.4,124.4,128.1,129.2,130.0,132.6$, 134.3, 138.7, 140.8, 148.3, 151.1, 153.5, $190.3(\mathrm{C}=\mathrm{O}), 192.8(\mathrm{C}=\mathrm{O})$; MS: $m / z=408.43$ [M] ${ }^{+}$; Analysis: for $\mathrm{C}_{21} \mathrm{H}_{20} \mathrm{~N}_{4} \mathrm{O}_{5}$, calcd. C 61.76, H 4.94, N 13.72\%; found C 61.90, H 4.92, N 13.77\%.

5-[4-(Morpholin-4-yl)benzoyl]-4-(4-nitrophenyl)-3,4-dihydropyrimidin-2(1H)-one (13): Yield: 70\%; m.p.: 202-204 ${ }^{\circ} \mathrm{C} ;{ }^{1} \mathrm{H}$ NMR $\left(500 \mathrm{MHz}, \mathrm{DMSO}-d_{6}\right): \delta=9.42\left(1 \mathrm{H}, \mathrm{d}, \mathrm{NH}, \mathrm{D}_{2} \mathrm{O}\right.$ exch), $8.23(1 \mathrm{H}, \mathrm{s},=\mathrm{CH})$, 7.43-7.92 (6H, m, Ar-H), $7.09\left(1 \mathrm{H}, \mathrm{s}, \mathrm{NH}, \mathrm{D}_{2} \mathrm{O}\right.$ exch), $6.94(2 \mathrm{H}, \mathrm{d}, J=8.9 \mathrm{~Hz}, \mathrm{Ar}-\mathrm{H}), 5.57(1 \mathrm{H}, \mathrm{s}$, $\mathrm{H}-4), 3.72\left(4 \mathrm{H}, \mathrm{t}, J=4.6 \mathrm{~Hz}, 2 \times \mathrm{CH}_{2}\right.$ morpholine $), 3.21\left(4 \mathrm{H}, \mathrm{t}, J=4.7 \mathrm{~Hz}, 2 \times \mathrm{CH}_{2}\right.$ morpholine $) ;{ }^{13} \mathrm{C} \mathrm{NMR}$ $\left(125.76 \mathrm{MHz}, \mathrm{DMSO}-d_{6}\right): \delta=47.60\left(\mathrm{CH}_{2}\right), 46.67\left(\mathrm{CH}_{2}\right), 53.9(\mathrm{CH}), 66.2\left(\mathrm{CH}_{2}\right), 66.3\left(\mathrm{CH}_{2}\right), 111.8,113.8$, 124.3, 128.2, 128.3, 130.5, 134.0, 138.0, 140.7, 147.2, 151.7, 151.8, 153.6, 190.5 (C=O), 192.0 (C=O); MS: $m / z=408.42[\mathrm{M}]^{+}$; Analysis: for $\mathrm{C}_{21} \mathrm{H}_{20} \mathrm{~N}_{4} \mathrm{O}_{5}$, calcd. C 61.76, $\mathrm{H} 4.94, \mathrm{~N} 13.72 \%$; found C 61.90, H 4.92, $\mathrm{N} 13.76 \%$.

5-[4-(Morpholin-4-yl)benzoyl]-4-(3-nitrophenyl)-3,4-dihydropyrimidin-2(1H)-one (14): Yield: 70\%; m.p.: 205-207 ${ }^{\circ} \mathrm{C} ;{ }^{1} \mathrm{H}$ NMR $\left(500 \mathrm{MHz}, \mathrm{DMSO}-d_{6}\right): \delta=9.40\left(1 \mathrm{H}, \mathrm{d}, \mathrm{NH}, \mathrm{D}_{2} \mathrm{O}\right.$ exch), $8.15(1 \mathrm{H}, \mathrm{s},=\mathrm{CH})$, 7.45-7.95 (6H, m, Ar-H), $7.12\left(1 \mathrm{H}, \mathrm{s}, \mathrm{NH}, \mathrm{D}_{2} \mathrm{O}\right.$ exch), $6.95(2 \mathrm{H}, \mathrm{d}, J=8.9 \mathrm{~Hz}, \mathrm{Ar}-\mathrm{H}), 5.59(1 \mathrm{H}, \mathrm{s}, \mathrm{H}-4)$, $3.72\left(4 \mathrm{H}, \mathrm{t}, J=4.6 \mathrm{~Hz}, 2 \times \mathrm{CH}_{2}\right.$ morpholine $), 3.22\left(4 \mathrm{H}, \mathrm{t}, J=4.7 \mathrm{~Hz}, 2 \times \mathrm{CH}_{2}\right.$ morpholine $) ;{ }^{13} \mathrm{C}$ NMR (125.76 $\left.\mathrm{MHz}, \mathrm{DMSO}-d_{6}\right): \delta=47.6\left(2 \times \mathrm{CH}_{2}\right), 53.7(\mathrm{CH}), 66.3\left(2 \times \mathrm{CH}_{2}\right), 111.8,113.8,121.6,122.9,128.2,130.5$, 130.7, 133.7, 140.8, 146.7, 148.2, 151.6, 153.6, $190.5(\mathrm{C}=\mathrm{O}), 192.0(\mathrm{C}=\mathrm{O})$; MS: $m / z=408.41[\mathrm{M}]^{+}$; Analysis: for $\mathrm{C}_{21} \mathrm{H}_{20} \mathrm{~N}_{4} \mathrm{O}_{5}$, calcd. C 61.76, H 4.94, N 13.72\%; found C61.89, H 4.91, N 13.75\%. 
4-(4-Chlorophenyl)-5-[4-(morpholin-4-yl)benzoyl]-3,4-dihydropyrimidin-2(1H)-one (15): Yield: 80\%; m.p.: $288-290{ }^{\circ} \mathrm{C} ;{ }^{1} \mathrm{H}$ NMR $\left(500 \mathrm{MHz}, \mathrm{DMSO}-d_{6}\right): \delta=9.25\left(1 \mathrm{H}, \mathrm{d}, \mathrm{NH}, \mathrm{D}_{2} \mathrm{O}\right.$ exch), $7.81(1 \mathrm{H}, \mathrm{s},=\mathrm{CH}), 7.33-7.45$ $(6 \mathrm{H}, \mathrm{m}, \mathrm{Ar}-\mathrm{H}), 7.02\left(1 \mathrm{H}, \mathrm{s}, \mathrm{NH}, \mathrm{D}_{2} \mathrm{O}\right.$ exch), $6.95(2 \mathrm{H}, \mathrm{d}, J=8.5 \mathrm{~Hz}, \mathrm{Ar}-\mathrm{H}), 5.43(1 \mathrm{H}, \mathrm{s}, \mathrm{H}-4), 3.73(4 \mathrm{H}$, $\mathrm{t}, J=4.6 \mathrm{~Hz}, 2 \times \mathrm{CH}_{2}$ morpholine $), 3.22\left(4 \mathrm{H}, \mathrm{t}, J=4.7 \mathrm{~Hz}, 2 \times \mathrm{CH}_{2}\right.$ morpholine $) ;{ }^{13} \mathrm{C} \mathrm{NMR}(125.76 \mathrm{MHz}$, DMSO- $\left.d_{6}\right): \delta=47.6\left(2 \times \mathrm{CH}_{2}\right), 53.6(\mathrm{CH}), 66.3\left(2 \times \mathrm{CH}_{2}\right), 112.5,113.8,128.4,128.8,128.9,130.5,132.3$, 140.1, 143.6, 151.8, 153.5, $190.6(\mathrm{C}=\mathrm{O}), 192.0(\mathrm{C}=\mathrm{O})$; $\mathrm{MS}: \mathrm{m} / \mathrm{z}=397.86[\mathrm{M}]^{+}$; Analysis: for $\mathrm{C}_{21} \mathrm{H}_{20} \mathrm{ClN}_{3} \mathrm{O}_{3}$, calcd. C 63.40, H 5.07, N 10.56\%; found C 63.65, H 5.08, N 10.59\%.

4-(2-Methoxyphenyl)-5-[4-(morpholin-4-yl)benzoyl]-3,4-dihydropyrimidin-2(1H)-one (16): Yield: 80\%; m.p.: 178-180 ${ }^{\circ} \mathrm{C} ;{ }^{1} \mathrm{H}$ NMR $\left(500 \mathrm{MHz}, \mathrm{DMSO}-d_{6}\right): \delta=9.22\left(1 \mathrm{H}, \mathrm{s}, \mathrm{NH}, \mathrm{D}_{2} \mathrm{O}\right.$ exch), $7.81(1 \mathrm{H}, \mathrm{s},=\mathrm{CH}), 7.20-7.50$ $(5 \mathrm{H}, \mathrm{m}, \mathrm{Ar}-\mathrm{H}), 7.09\left(1 \mathrm{H}, \mathrm{s}, \mathrm{NH}, \mathrm{D}_{2} \mathrm{O}\right.$ exch), 6.89-7.01 $(3 \mathrm{H}, \mathrm{m}, \mathrm{Ar}-\mathrm{H}), 5.75(1 \mathrm{H}, \mathrm{s}, \mathrm{H}-4), 3.82(4 \mathrm{H}, \mathrm{t}$, $J=4.7 \mathrm{~Hz}, 2 \times \mathrm{CH}_{2}$ morpholine), $3.21\left(4 \mathrm{H}, \mathrm{t}, J=4.7 \mathrm{~Hz}, 2 \times \mathrm{CH}_{2}\right.$ morpholine $) ;{ }^{13} \mathrm{C} \mathrm{NMR}(125.76 \mathrm{MHz}$, DMSO- $\left.d_{6}\right): \delta=47.6\left(2 \times \mathrm{CH}_{2}\right), 49.6\left(\mathrm{OCH}_{3}\right), 55.9(\mathrm{CH}), 66.2\left(\mathrm{CH}_{2}\right), 66.3\left(\mathrm{CH}_{2}\right), 111.5,117.7,113.8,120.7$, 127.9, 128.7, 129.3, 130.5, 131.3, 140.4, 152.2, 153.5, 157.3, 190.5 (C=O), 192.0 (C=O); MS: $m / z=393.41$ $[\mathrm{M}]^{+}$; Analysis: for $\mathrm{C}_{22} \mathrm{H}_{23} \mathrm{~N}_{3} \mathrm{O}_{4}$, calcd. C 67.16, H 5.89, N 10.68\%; found C 66.89, H 5.87, N 10.64\%.

4-(4-Hydroxyphenyl)-5-[4-(morpholin-4-yl)benzoyl]-3,4-dihydropyrimidin-2(1H)-one (17): Yield: 60\%; m.p.: 118-120 ${ }^{\circ} \mathrm{C} ;{ }^{1} \mathrm{H}$ NMR (500 MHz, DMSO- $\left.d_{6}\right): \delta=9.14\left(1 \mathrm{H}, \mathrm{s}, \mathrm{NH}, \mathrm{D}_{2} \mathrm{O}\right.$ exch), $9.01(1 \mathrm{H}, \mathrm{s}, \mathrm{OH}), 8.08(1 \mathrm{H}$, $\mathrm{s},=\mathrm{CH}), 7.43-7.77(4 \mathrm{H}, \mathrm{m}, \mathrm{Ar}-\mathrm{H}), 7.07\left(1 \mathrm{H}, \mathrm{s}, \mathrm{NH}, \mathrm{D}_{2} \mathrm{O}\right.$ exch), 6.70-7.05 $(4 \mathrm{H}, \mathrm{m}, \mathrm{Ar}-\mathrm{H}), 5.35(1 \mathrm{H}, \mathrm{s}$, $\mathrm{H}-4), 3.74\left(4 \mathrm{H}, \mathrm{t}, J=4.6 \mathrm{~Hz}, 2 \times \mathrm{CH}_{2}\right.$ morpholine $), 3.21\left(4 \mathrm{H}, \mathrm{t}, J=4.6 \mathrm{~Hz}, 2 \times \mathrm{CH}_{2}\right.$ morpholine $) ;{ }^{13} \mathrm{C} \mathrm{NMR}$ $\left(125.76 \mathrm{MHz}, \mathrm{DMSO}-d_{6}\right): \delta=47.0\left(\mathrm{CH}_{2}\right), 47.6\left(\mathrm{CH}_{2}\right), 53.5(\mathrm{CH}), 66.2\left(\mathrm{CH}_{2}\right), 66.4\left(\mathrm{CH}_{2}\right), 113.4,113.8$, 115.5, 128.0, 128.6, 130.5, 132.6, 135.2, 139.3, 152.0, 153.5, 154.5, 154.7, 190.7 (C=O), 191.8 (C=O); MS: $m / z=379.41[\mathrm{M}]^{+}$; Analysis: for $\mathrm{C}_{21} \mathrm{H}_{21} \mathrm{~N}_{3} \mathrm{O}_{4}$, calcd. C 66.48, H 5.58, N 11.08\%; found C 66.72, H 5.60, N $11.04 \%$.

4-(3-Hydroxyphenyl)-5-[4-(morpholin-4-yl)benzoyl]-3,4-dihydropyrimidin-2(1H)-one (18): Yield: 60\%; m.p.: 120-122 ${ }^{\circ} \mathrm{C} ;{ }^{1} \mathrm{H}$ NMR $\left(500 \mathrm{MHz}\right.$, DMSO- $\left.d_{6}\right): \delta=9.19\left(1 \mathrm{H}, \mathrm{s}, \mathrm{NH}, \mathrm{D}_{2} \mathrm{O}\right.$ exch), $9.01(1 \mathrm{H}, \mathrm{s}, \mathrm{OH}), 8.08(1 \mathrm{H}$, $\mathrm{s},=\mathrm{CH}), 7.43-7.77(4 \mathrm{H}, \mathrm{m}, \mathrm{Ar}-\mathrm{H}), 7.06\left(1 \mathrm{H}, \mathrm{s}, \mathrm{NH}, \mathrm{D}_{2} \mathrm{O}\right.$ exch $), 6.63-7.01(4 \mathrm{H}, \mathrm{m}, \mathrm{Ar}-\mathrm{H}), 5.37(1 \mathrm{H}, \mathrm{s}$, $\mathrm{H}-4), 3.72\left(4 \mathrm{H}, \mathrm{t}, J=4.6 \mathrm{~Hz}, 2 \times \mathrm{CH}_{2}\right.$ morpholine $), 3.20\left(4 \mathrm{H}, \mathrm{t}, J=4.6 \mathrm{~Hz}, 2 \times \mathrm{CH}_{2}\right.$ morpholine $) ;{ }^{13} \mathrm{C} \mathrm{NMR}$ $\left(125.76 \mathrm{MHz}, \mathrm{DMSO}-d_{6}\right): \delta=48.3\left(\mathrm{CH}_{2}\right), 48.5\left(\mathrm{CH}_{2}\right), 55.2(\mathrm{CH}), 66.7\left(\mathrm{CH}_{2}\right), 67.6\left(\mathrm{CH}_{2}\right), 114.6,115.0$, 131.2, 131.8, 138.9, 140.1, 140.8, 147.4, 153.4, 154.6, 154.8, 155.8, 159.2, 192.0 (C=O), 194.0 (C=O); MS: $m / z=379.42[\mathrm{M}]^{+}$; Analysis: for $\mathrm{C}_{21} \mathrm{H}_{21} \mathrm{~N}_{3} \mathrm{O}_{4}$, calcd. C 66.48, H 5.58, N 11.08\%; found C 66.63, H 5.59, $\mathrm{N} 11.12 \%$.

4-(3-Methoxyhenyl)-5-[4-(morpholin-4-yl)benzoyl]-3,4-dihydropyrimidin-2(1H)-one (19): Yield: 60\%; m.p.: 170-172 ${ }^{\circ} \mathrm{C} ;{ }^{1} \mathrm{H}$ NMR $\left(500 \mathrm{MHz}\right.$, DMSO- $\left.d_{6}\right): \delta=9.23\left(1 \mathrm{H}, \mathrm{d}, \mathrm{NH}, \mathrm{D}_{2} \mathrm{O}\right.$ exch), $8.09(1 \mathrm{H}, \mathrm{s},=\mathrm{CH}), 7.24-7.79$ $(4 \mathrm{H}, \mathrm{m}, \mathrm{Ar}-\mathrm{H}), 6.87\left(1 \mathrm{H}, \mathrm{s}, \mathrm{NH}, \mathrm{D}_{2} \mathrm{O}\right.$ exch), 6.91-7.04 $(4 \mathrm{H}, \mathrm{m}, \mathrm{Ar}-\mathrm{H}), 5.45(1 \mathrm{H}, \mathrm{s}, \mathrm{H}-4), 3.83(3 \mathrm{H}, \mathrm{s}$, $\left.\mathrm{OCH}_{3}\right), 3.73\left(4 \mathrm{H}, \mathrm{t}, J=4.6 \mathrm{~Hz}, 2 \times \mathrm{CH}_{2}\right.$ morpholine), $3.22\left(4 \mathrm{H}, \mathrm{t}, J=4.6 \mathrm{~Hz}, 2 \times \mathrm{CH}_{2}\right.$ morpholine); ${ }^{13} \mathrm{C} \mathrm{NMR}$ $\left(125.76 \mathrm{MHz}, \mathrm{DMSO}-d_{6}\right): \delta=47.0\left(\mathrm{CH}_{2}\right), 47.6\left(\mathrm{CH}_{2}\right), 53.9\left(\mathrm{OCH}_{3}\right), 55.4(\mathrm{CH}), 66.2\left(\mathrm{CH}_{2}\right), 66.3\left(\mathrm{CH}_{2}\right)$, 112.77, 112.79, 112.93, 113.8, 118.9, 128.5, 130.1, 130.5, 139.8, 146.1, 152.0, 153.5, 159.7, 190.7 (C=O), 192.0 (C=O); MS: $m / z=393.40[\mathrm{M}]^{+}$; Analysis: for $\mathrm{C}_{22} \mathrm{H}_{23} \mathrm{~N}_{3} \mathrm{O}_{4}$, calcd. C 67.16, $\mathrm{H} 5.89, \mathrm{~N} 10.68 \%$; found C 66.87, H 5.91, N 10.66\%.

4-(4-Ethoxyphenyl)-5-[4-(morpholin-4-yl)benzoyl]-3,4-dihydropyrimidin-2(1H)-one (20): Yield: 60\%; m.p.: 200-202 ${ }^{\circ} \mathrm{C}^{1}{ }^{1} \mathrm{H}$ NMR $\left(500 \mathrm{MHz}\right.$, DMSO- $\left.d_{6}\right): \delta=9.16\left(1 \mathrm{H}, \mathrm{s}, \mathrm{NH}, \mathrm{D}_{2} \mathrm{O}\right.$ exch), $8.08(1 \mathrm{H}, \mathrm{s},=\mathrm{CH}), 6.86-7.77$ $(8 \mathrm{H}, \mathrm{m}, \mathrm{Ar}-\mathrm{H}), 6.78\left(1 \mathrm{H}, \mathrm{s}, \mathrm{NH}, \mathrm{D}_{2} \mathrm{O}\right.$ exch), $5.38(1 \mathrm{H}, \mathrm{s}, \mathrm{H}-4), 3.97\left(2 \mathrm{H}, \mathrm{q}, J=6.9 \mathrm{~Hz}, \mathrm{OCH}_{2}\right), 3.73(4 \mathrm{H}, \mathrm{t}$, $J=4.6 \mathrm{~Hz}, 2 \times \mathrm{CH}_{2}$ morpholine), $3.21\left(4 \mathrm{H}, \mathrm{t}, J=4.6 \mathrm{~Hz}, 2 \times \mathrm{CH}_{2}\right.$ morpholine $), 1.27\left(3 \mathrm{H}, \mathrm{t}, J=6.9 \mathrm{~Hz}, \mathrm{CH}_{3}\right)$; ${ }^{13} \mathrm{C}$ NMR $\left(125.76 \mathrm{MHz}, \mathrm{DMSO}-d_{6}\right): \delta=18.0\left(\mathrm{CH}_{3}\right), 47.0\left(\mathrm{CH}_{2}\right), 47.7\left(\mathrm{CH}_{2}\right), 53.4\left(\mathrm{OCH}_{2}\right), 63.2\left(\mathrm{CH}_{2}\right)$, $66.3\left(\mathrm{CH}_{2}\right), 113.2,114.2,125.8,128.0,129.5,132.7,136.7,138.8,139.5,151.9,153.3,154.6,157.1,158.2$, $190.7(\mathrm{C}=\mathrm{O}), 192.8(\mathrm{C}=\mathrm{O})$; MS: $m / z=407.42[\mathrm{M}]^{+}$; Analysis: for $\mathrm{C}_{23} \mathrm{H}_{25} \mathrm{~N}_{3} \mathrm{O}_{4}$, calcd. C 67.80, H 6.18, $\mathrm{N}$ $10.31 \%$; found C $66.88, \mathrm{H} 6.20, \mathrm{~N} 10.35 \%$. 


\section{Conclusions}

In conclusion, novel dihydropyrimidinone derivatives (1-20) containing piperazine and morpholine moieties were synthesized efficiently in good yield with a simple method consisting of three components in a single pot. The starting material, enaminones, 4-methyl-1-[4(piperazin/morpholin-1-yl) phenyl] pent-2-en-1-one (IIa-b) were synthesized by reacting 4-methyl-1[4-(piperazin/morpholin-1-yl) phenyl] pent-2-en-1-one (Ia-b) with dimethylformamide dimethylacetal (DMF-DMA) without solvent. The $E$-configuration of the enaminone was confirmed by the single crystal X-ray crystallography.

Author Contributions: Data curation, A.M.N.; Formal analysis, H.A.G.; Investigation, M.A.B.; Project administration, M.A.A.-O.

Funding: The authors would like to extend their sincere appreciation to the Deanship of Scientific Research at King Saud University for funding this research group no. (RG 1435-006).

Conflicts of Interest: The authors declare no conflict of interests.

\section{References}

1. Folkers, K.; Harwood, H.J.; Johnson, T.B. Researches on pyrimidines. cxxx. Synthesis of 2-keto-1,2,3,4tetrahydropyrimidines. J. Am. Chem. Soc. 1932, 54, 3751-3758. [CrossRef]

2. Atwal, K.S.; Ahmed, S.Z.; Bird, J.E.; Delaney, C.L.; Dickinson, K.E.; Ferrara, F.N.; Hedberg, A.; Miller, A.V.; Moreland, S.; O'Reilly, B.C.; et al. Dihydropyrimidine angiotensin II receptor antagonists. Dihydropyrimidine calcium channel blockers. 3. 3-Carbamoyl-4-aryl-1,2,3,4-tetrahydro-6-methyl-5-pyrimidinecarboxylic acid esters as orally effective antihypertensive agents. J. Med. Chem. 1992, 35, 4751-4763. [CrossRef] [PubMed]

3. Rovnyak, G.C.; Kimball, S.D.; Beyer, B.; Cucinotta, G.; DiMarco, J.D.; Gougoutas, J.; Hedberg, A.; Malley, M.; McCarthy, J.P.; Zhang, R.; et al. Calcium entry blockers and activators: Conformational and structural determinants of dihydropyrimidine calcium channel modulators. J. Med. Chem. 1995, 38, 119-129. [CrossRef] [PubMed]

4. Rana, K.; Kaur, B.; Kumar, B. Synthesis and antihypertensive activity of some dihydropyrimidines. Ind. J. Chem. 2004, 43, 1553-1557. [CrossRef]

5. Beena, K.P.; Suresh, R.; Rajasekaran, A.; Manna, P.K. DihydroPyrimidinones-A Versatile Scaffold with Diverse Biological Activity. J. Pharm. Sci. Res. 2016, 8, 741-746.

6. Bhat, M.A.; Al-Dhfyan, A.; Al-Omar, M.A. Targeting Cancer Stem Cells with Novel 4-(4-2substitutedphenyl)-5-(3,4,5-trimethoxy/3,4-dimethoxy)-3benzoyl-3,4-ihydropyrimidine-2(1H)-one/thiones. Molecules 2016, 21, 1746. [CrossRef] [PubMed]

7. Atwal, K.S.; Swanson, B.N.; Unger, S.E.; Floyd, D.M.; Moreland, S.; Hedberg, A.; O'Reilly, B.C. Dihydropyrimidine calcium channel blockers. 3. 3-Carbamoyl-4-aryl-1,2,3,4-tetrahydro-6-methyl-5-pyrimidinecarboxylic acid esters as orally effective antihypertensive agents. J. Med. Chem. 1991, 34, 806-811. [CrossRef] [PubMed]

8. Kappe, C.O. 100 Years of the Biginelli dihydropyridine synthesis. Tetrahedron 1993, 49, 6937-6963. [CrossRef]

9. Al-Ghorbani, M.; Bushra, B.A.; Zabiulla, M.S.V.; Mamatha, S.V.; Khanum, S.A. Piperazine and morpholine: Synthetic preview and pharmaceutical applications. J. Chem. Pharm. Res. 2015, 7, 281-301. [CrossRef]

10. Patel, R.V.; Park, S.W. An evolving role of piperazine moieties in drug design and discovery. Mini-Rev. Med. Chem. 2013, 13, 1579-1601. [CrossRef] [PubMed]

11. Lopez-Rodriguez, M.L.; Ayala, D.; Benhamu, B.; Morcillo, M.J.; Viso, A. Arylpiperazine derivatives acting at 5-HT(1A) receptors. Curr. Med. Chem. 2002, 9, 443-469. [CrossRef] [PubMed]

12. Lacivita, E.; Leopoldo, M.; De Giorgio, P.; Berardi, F.; Perrone, R. Determination of 1-aryl-4-propylpiperazine pKa values: The substituent on aryl modulates basicity. Bioorg. Med. Chem. 2009, 17, 1339-1344. [CrossRef] [PubMed]

13. Haupt, A.; Pohlki, F.; Drescher, K.; Wicke, K.; Unger, L.; Relo, A.; Bespalov, A.; Vogg, B.; Backfisch, G.; Delzer, J.; et al. N-Phenyl-(piperazinyl or homopiperazinyl)-benzenesulfonamide or Benzenesulfonylphenyl-(piperazine or homopiperazine) Compounds Suitable for Treating Disorders that Respond to Modulation of the Serotonin 5-HT-6 Receptor. WO 2010125134 A1, 4 November 2010. 
14. Freire, T.R.V.; Mannochio, D.S.R.E. Compounds and Pharmaceutical Compositions for Treating Disorders Associated with the 5-HT1a and 5-HT2a Receptors. WO 2012037634 A1, 29 March 2012.

15. Højer, A.M.; Drewes, P.G.; Kateb, J. New Compositions of 1-[2-(2,4-dimethyl-phenylsulfanyl)-phenyl]piperazine. WO 2011023194 A2, 3 March 2011.

16. Ito, N.; Sasaki, H.; Tai, K.; Shinohara, T. Heterocyclic Compounds for Treating or Preventing Disorders Caused by Reduced Neurotransmission of Serotonin, Norephnephrine or Dopamine. WO 2012036253 A1, 22 March 2012.

17. Bang-Andersen, B.; Mork, A.; Moore, N.; Stensbol, T. 1-[2-(2,4-Dimethylphenylsulfanyl)-phenyl]piperazine as a Compound with Combined Serotonin Reuptake, 5-HT3 and 5-HT1a Activity for the Treatment of Cognitive Impairment. U.S. Patent 20140248355 A1, 4 September 2014.

18. Zhang, Y.; Jin, C.; Zhou, R. Preparation of Pyrimidinyl Piperazine Derivatives as Selective Serotonin Reuptake Inhibitors for the Treatment and Prevention of CNS Disorders. WO 2015014256 A1, 5 February 2015.

19. Achanath, R.; Jose, J.; Rangaswamy, C.; Mandal, S.; Balaji, S.; Kadavilpparampulan, M.A.M.; Newington, I.M. Preparation of Piperazine Derivatives as Imaging Agents. WO 2013041682 A1, 28 March 2013.

20. Hoenke, C.; Giovannini, R.; Lessel, U.; Rosenbrock, H.; Schmid, B. Piperazine Derivatives and the Use of Thereof as a Medicament. WO 2015055698 A1, 23 April 2015.

21. Jin, L.; Yang, R.; Song, R.; Zhu, H.; Wu, N.; Yun, L.; Su, R.; Zhao, R. Preparation of Novel Piperazine Derivatives as Dopamine D3 Receptor Ligands. U.S. Patent 2014/0329831 A1, 6 November 2014.

22. Ganesh, T.; Sun, A.; Smith, S.M.; Lambeth, J.D. Preparation of Piperazine Derivatives for Use as NADPH-Oxidase Inhibitors. WO 2012173952 A1, 20 December 2012.

23. Yeung, K.S.; Farkas, M.E.; Kadow, J.F.; Meanwell, N.A.; Taylor, M.; Johnston, D.; Coulter, T.; Wright, J.J. Indole, Azaindole and Related Heterocyclic N-Substituted Piperazine Derivatives and Their Preparation and Use for the Treatment of HIV Infection. U.S. Patent 8039486 B2, 18 October 2011.

24. Sofia, M.J.; Kakarla, R.; Liu, J.; Naduthambi, D.; Mosley, R.; Steuer, H.M. Preparation of Piperazine Derivatives and Their Uses to Treat Viral Infections, Including Hepatitis C. U.S. Patent 20120202794 A1, 9 August 2012.

25. Carniato, D.; Briand, J.F.; Gutmann, M.; Busnel, O.; Bougeret, C.; Deprez, B.; Jaillardon, K. Preparation of Piperazine Derivatives Useful for the Treatment of Cancers, Especially Cancers Resistant to Chemotherapy. WO 2013098393 A1, 4 July 2013.

26. Wang, S.; Zhou, H.; Chen, J.; Aguilar, A.; Meagher, J.L.; Sun, D.; Yang, C.; Liu, L.; Bai, L.; McEachem, D.; et al. Synthesis of Piperazine Derivatives as Bcl-2/Bcl-xL Inhibitors. U.S. Patent 20120189539 A1, 26 July 2012.

27. Csonka, I.; Grolmusz, V.; Répási, J.; Szabadka, Z.; Szabo, A.; Kertesz, M. Piperazine Derivatives and Their Preparation and Use as Tuberculostatics. WO 2011089456 A1, 28 July 2011.

28. Keliher, E.J.; Reiner, T.; Weissleder, R. Preparation of Substituted Piperazine Derivatives for Use in Targeting PARP-1 for Detection and Imaging of Cancer. WO 2012074840 A2, 7 June 2012.

29. Kato, S.; Morie, T.; Hino, K.; Kon, T.; Naruto, S.; Yoshida, N.; Karasawa, T.; Matsumoto, J. Novel benzamides as selective and potent gastric prokinetic agents. 1. Synthesis and structure-activity relationships of N-[(2-Morpholinyl)alkyl]benzamides. J. Med. Chem. 1990, 33, 1406-1413. [CrossRef] [PubMed]

30. Chrysselis, M.C.; Rekka, E.A.; Kourounakis, P.N. Hypocholesterolemic and hypolipidemic activity of some novel morpholine derivatives with antioxidant Activity. J. Med. Chem. 2000, 43, 609-612. [CrossRef] [PubMed]

31. Hale, J.J.; Mills, S.G.; MacCoss, M.; Dorn, C.P.; Finke, P.E.; Budhu, R.J.; Reamer, R.A.; Huskey, S.E.; Luffer-Atlas, D.; Dean, B.J.; et al. Phosphorylated morpholine acetal human neurokinin-1 receptor antagonists as water-soluble prodrugs. J. Med. Chem. 2000, 43, 1234-1241. [CrossRef] [PubMed]

32. Kuettel, S.; Zambon, A.; Kaiser, M.; Brun, R.; Scapozza, L.; Perozzo, R. Synthesis and evaluation of antiparasitic activities of new 4-[5-(4-Phenoxyphenyl)-2H-pyrazol-3-yl]morpholine derivatives. J. Med. Chem. 2007, 50, 5833-5839. [CrossRef] [PubMed]

33. Takaya, M.; Sato, M.; Terashima, K.; Tanizawa, H.; Maki, Y. A new Nonsteroidal analgesic-anti-inflammatory Agent. Synthesis and activity of 4-Ethoxy-2-methyl-5-morpholino-3(2H)-pyridazinone and related compounds. J. Med. Chem. 1979, 22, 53-58. [CrossRef] [PubMed]

34. Araki, K.; Kuroda, T.; Uemori, S.; Moriguchi, A.; Ikeda, Y.; Hirayama, F.; Yokoyama, Y.; Iwao, E.; Yakushiji, T. Quinolone antimicrobial agents substituted with morpholines at the 7-Position, synthesis and structure-activity relationships. J. Med. Chem. 1993, 36, 1356-1363. [CrossRef] [PubMed] 
35. Lukas, R.J.; Muresan, A.Z.; Damaj, M.I.; Blough, B.E.; Huang, X.; Navarro, H.A.; Mascarella, S.W.; Eaton, J.B.; Marxer-Miller, S.K.; Carroll, F.I. Synthesis and characterization of in vitro and in vivo profiles of hydroxybupropion analogues: Aids to smoking cessation. J. Med. Chem. 2010, 53, 4731-4748. [CrossRef] [PubMed]

36. Bhat, M.A.; Ahmed, A.F.; Wen, Z.H.; Al-Omar, M.A.; Abdel-Aziz, H.A. Synthesis, anti-inflammatory and neuroprotective activity of pyrazole and pyrazolo[3,4-d]pyridazine bearing 3,4,5-trimethoxyphenyl. Med. Chem. Res. 2017, 26, 1557-1566. [CrossRef]

37. Bhat, M.A.; Al-Rashood, K.A.; Abdel-Aziz, H.A. Unexpected configuration in stereoslective synthesis of some novel (1Z)-1-(morpholin-1-yl)-N2-arylamidrazones. Lett. Org. Chem. 2012, 9, 487-492. [CrossRef]

38. Bhat, M.A.; Al-Omar, M.A.; Naglah, A.M. Synthesis and in vivo anti-ulcer evaluation of some novel piperidine linked dihydropyrimidinone derivatives. J. Enzym. Inhib. Med. Chem. 2018, 33, 978-988. [CrossRef] [PubMed]

39. Al-Dhfyan, A.; Bhat, M.A. Method for Treating Cancer Using a Dihydropyrimidine Derivative. U.S. Patent 9,119,856 B1, 1 September 2015.

40. Allen, F.H.; Kennard, O.; Watson, D.G.; Brammer, L.; Orpen, A.G.; Taylor, R. Tables of bond lengths determined by X-ray and neutron diffraction. Part 1. Bond lengths in organic compounds. J. Chem. Soc. Perkins Trans. 1987, 12, S1-S19. [CrossRef]

41. Sheldrick, G.M. A short history of SHELX. Acta Crystallogr. A 2008, 64, 112-122. [CrossRef] [PubMed]

42. Sheldrick, G.M. SHELXTL-PC, version 5.1; Siemens Analytical Instruments, Inc.: Madison, WI, USA, 1997.

Sample Availability: Samples of the compounds (1-20) with 99\% purity are available from authors.

(C) 2018 by the authors. Licensee MDPI, Basel, Switzerland. This article is an open access article distributed under the terms and conditions of the Creative Commons Attribution (CC BY) license (http:// creativecommons.org/licenses/by/4.0/). 